\title{
Lipschitz Spaces and the Heat Equation
}

\author{
B. FRANK JONES, JR.*
}

\author{
Communicated by Jurgen Moser
}

1. Introduction. Several authors have now treated a wide variety of classes of function spaces. For example, the survey article [10] contains some very general results on Lipschitz spaces of functions of $n$ variables with smoothness conditions which can be different in each of the $n$ coordinate directions. In case the smoothness conditions are rotationally invariant, there are intimate connections between the function spaces which arise and the properties of solutions of Laplace's equation in the half space $\mathrm{R}^{n} \times(0, \infty)$ with boundary values in the given function spaces. See [15], [16], [17] for a detailed exposition of these properties. The purpose of this paper is to obtain the similar development in case the function spaces have one coordinate treated differently than the others, and to relate certain of these spaces to solutions of the heat equation in a half space. In anticipation of this paper some results in this direction have already been given [9], which mainly involve the theory of singular integral operators with mixed homogeneity [1], [4], [8].

In fact, the key to almost everything in this paper is the mixed homogeneity. The fact that solutions of the heat equation $u_{t}=u_{x_{1} x_{1}}+\ldots+u_{x_{n} x_{n}}+u_{y y}$ are invariant under the transformations $x \rightarrow \lambda x, y \rightarrow \lambda y, t \rightarrow \lambda^{2} t$, indicates that changes of variables of this form should be used in the analysis. Therefore, expressions for norms etc. should be so constructed as to be invariant under such transformations. Thus, for example, the measure $\left(|x|+|t|^{1 / 2}\right)^{-n-2} d x d t$ appears frequently.

Since the motivation for this paper arises from the desire to correlate smoothness properties of functions which are to act as boundary values for solutions of the heat equation with the behavior of the solutions near the boundary, we have taken the somewhat unusual approach of considering the pure boundary value problem-i.e., no initial condition is to be assigned. Thus, the solutions of the heat equation in this paper will always exist for $-\infty<t<\infty$, rather than the usual $0<t<\infty$. It is trivial to observe that if the boundary values happen to vanish for $t<0$, then the solution can be viewed as a solution of the mixed problem with boundary values specified for $t>0$ and initial values (at $t=0$ ) identically zero.

\footnotetext{
* Research sponsored by the Air Force Office of Scientific Research, Office of Aerospace Research, United States Air Force, under AFOSR Grant No. 941-65.
} 
The elimination of initial conditions from the problem necessitates the derivation of uniqueness theorems which are probably known but not standard; consequently, we have included in Lemmas 2-4 complete proofs of the relevant uniqueness statements. Here a sharp distinction arises between the two types of problems. For the mixed problem the stabilizing condition on the solution can be as weak as requiring the solution to be bounded by a constant multiple of $\exp \left(C\left(|x|^{2}+y^{2}\right)\right)$ for any $C>0$. However, the corresponding result in our case (Lemma 2) has the hypothesis that the solution be bounded by a constant; this could be improved only slightly, as the counter-example $u(x, t ; y) \equiv y$ shows.

We now indicate the main ideas in the paper. Section 2 contains preliminary material, including the uniqueness results mentioned above. Already in this section the situation of mixed homogeneity is seen, especially in Lemma 5 and the definition preceding it. Briefly, the reason is as follows. In treating the generalized boundary value problem in which the "boundary values" can be a measure $\mu$, one is led to a discussion of differentiation of $\mu$ with respect to Lebesgue measure. The most well known theories of differentiation of a measure require a condition that the sets used to define the derivative of $\mu$ at a point $P$ not only converge to $P$, but in such a manner that they do not become too thin in the process; e.g., that each of the sets is contained in a cube centered at $P$ whose volume is bounded by a constant multiple of the Lebesgue measure of the set in question. However, such a theory cannot be applied in this case, because cubes in the $x-t$ space $\mathrm{R}^{n+1}$ do not possess the requisite homogeneity. So "cubes" must be replaced by sets of the form $\left\{(z, s) \varepsilon \mathrm{R}^{n+1}:|z-x|<a,|s-t|<a^{2}\right\}$, where $z, x \varepsilon \mathrm{R}^{n}$ and $|z-x|$ is the Euclidean norm. These sets obviously become "thinner" in the $t$-direction than in the $x$-direction. Fortunately, the standard theory of differentiation has a simple modification to allow this case to be handled easily [3], [7].

Along the same lines, the "non-tangential" limiting behavior of solutions, which has been thoroughly discussed in many situations, must be modified since Euclidean cones in the $x-t-y$ space $\mathrm{R}^{n+2}$ do not have the correct homogeneity. Instead, the set $P_{\alpha}(x, t)$ of Lemma 5 and Theorem 1 is the correct replacement for a cone in $\mathrm{R}^{n+2}$ with vertex $(x, t)$. The generality of Lemma 5 and Theorem 1 is really more than we require, as orthogonal approach to the boundary would really suffice, but the general results have been included both for the sake of completeness and to illustrate fully the homogeneity principles involved.

The remainder of Section 2 consists of collecting several of the important technical tools to be used in the following sections.

Section 3 contains the main results on the Lipschitz spaces, which are defined in terms of the growth properties as $y \rightarrow 0$ of $D_{v}^{k} u$, where $u$ is the solution of the boundary value problem for the heat equation $u_{t}=u_{x_{1} x_{1}}+\ldots+u_{x_{n} x_{n}}+u_{y y}$ for $y>0$ with boundary values $u=f(x, t)$ for $y=0$. The definition seems rather arbitrary, except that it is an exact analog of the corresponding definition of Taibleson [15]. Its value lies in the fact that the only distinction made between $x_{i}$ and $t$ occurs in the measure defining the $p q$ norm used (cf. (1.2)). However, 
the main theorem of Section 3 (Theorem 3) exhibits explicitly the different roles played by $x_{i}$ and $t$. For $0<\alpha<2$ this theorem characterizes the Lipschitz spaces in a way which does not even involve the heat equation, and thus avoids mention of the kernel $\Gamma$. A very rough description of the result would be that for a function $f(x, t)$ to be in a particular Lipschitz space it is necessary and sufficient that it have a certain very precise smoothness property, describable in terms of difference quotients of $f$. This incidentally justifies the terminology "Lipschitz space." In turn, this smoothness property of $f$ is necessary and sufficient for the solution of the heat equation with boundary values $f$ to converge to $f$ as $y \rightarrow 0$ at a precise rate.

The corresponding results in [15] give an indication of how to proceed in the proof of our results. It should also be mentioned that Taibleson's development includes a discussion of the heat equation as well as Laplace's equation, but the differential problem he treats is the pure initial value problem. That is, he considers functions $f(x)$ defined for $x \varepsilon \mathrm{R}^{n}$ and solves the problem $u_{t}=u_{x_{1} x_{1}}+$ $\ldots+u_{x_{n} x_{n}}$ for $t>0$, with initial condition $u(x, 0)=f(x)$. Again, $x$ and $t$ have to be treated with the corresponding homogeneity, but this question can be eliminated by observing that the crucial homogeneity considerations take place in the boundary, in this case $t=0$. But $x_{1}, x_{2}, \ldots, x_{n}$ are all treated alike, so no special considerations are necessary. This becomes even more evident if one introduces a new variable $t=\tau^{2}$, changing the heat equation into $(2 \tau)^{-1} u_{\tau}=$ $u_{x_{1} x_{1}}+\ldots+u_{x_{n} x_{n}}$, and giving $\tau, x_{1}, \ldots, x_{n}$ the same homogeneity properties. This procedure was used in [6].

The rest of Section 3 contains properties of the Lipschitz spaces.

The results of Section 3 are not complete. The difficulties causing this situation are discussed in Section 3 and Section 5 , the latter containing the needed missing results in the simpler case $n=0$.

Section 4 contains the definition and properties of spaces related to the Lipschitz spaces, but defined by means of potentials. The main result here is Theorem 7, which establishes an isomorphism between the various Lipschitz spaces. Further results on the spaces defined in Section 4, including their relation to the Lipschitz spaces, are to be found in [9].

Analogs of many of the results in [15] have not been included here. One such omission is that of an explicit definition of spaces $\Lambda^{\prime}(\alpha ; p, q)$ for $\alpha \leqq 0$. Another is any mention of the behavior of these spaces under the operations of extension or restriction. Some results along this line can be obtained for certain values of $\alpha$, but our lack of an explicit characterization of $\Lambda^{\prime}(2 ; p, q)$ (cf. Section 5) obviates general results of this type. It is anticipated that a later paper will contain such results.

Added in proof. C. C. Tu and the author have obtained several extension and restriction theorems valid for the full range of $\alpha>0$.

The notation used in this paper will now be discussed. The basic spaces are spaces of functions of $n+1$ real variables. Always one of these variables, the "time," is distinguished and given a distinct notation. Thus, in speaking of a 
function $f$ on $\mathbf{R}^{n+1}$, the points in $\mathbf{R}^{n+1}$ are designated $(x, t),(z, s)$, etc., where $x \varepsilon \mathbf{R}^{n}, z \varepsilon \mathbf{R}^{n}$, etc., and the function is written $f$ or $f(x, t)$ or $f(z, s)$, etc. It is also frequently necessary to consider functions on $\mathrm{R}_{+}^{n+2}=\left\{(x, t, y):(x, t) \varepsilon \mathbf{R}^{n+1}\right.$, $0<y<\infty\}$. Such a function is written $u$ or $u(x, t ; y)$; if the discussion allows, this function will also be written $u(y)$, emphasizing that $y$ is fixed for the moment. The norms $L^{p}$ are always taken with respect to functions on $\mathrm{R}^{n+1}$ and denoted

$$
\begin{aligned}
& \|f\|_{p}=\left(\int_{\mathbf{R}^{n+1}}|f(x, t)|^{p} d x d t\right)^{1 / p}, \quad 1 \leqq p<\infty, \\
& \|f\|_{\infty}=\text { ess. sup. }|f(x, t)| .
\end{aligned}
$$

Thus, for example

$$
\|u(y)\|_{p}=\left(\int_{\mathbf{R}^{n+1}}|u(x, t ; y)|^{p} d x d t\right)^{1 / p}
$$

Convolution is always denoted $*$ and is always convolution on $\mathbf{R}^{n+1}$ : thus $\Gamma(y) * f$ is the function whose value at $(x, t)$ is

$$
\int_{\mathbf{R}^{n+1}} \Gamma(x-z, t-s ; y) f(z, s) d z d s .
$$

The Hölder conjugate of $p$ is denoted $p^{\prime}$.

The Euclidean inner product of $x$ and $z$ in $\mathrm{R}^{n}$ is denoted $x \cdot z ;|x|=(x \cdot x)^{1 / 2}$. The Laplace operator in $R^{n}$ is denoted $\Delta: \Delta=\partial^{2} / \partial x_{1}^{2}+\cdots+\partial^{2} / \partial x_{n}^{2}$.

The closure of $\mathbf{R}_{+}^{n+2}$ is denoted $\mathbf{R}_{+}^{\overline{n+2}}$.

The space of infinitely differentiable functions on $\mathrm{R}^{n+1}$ which together with their derivatives tend to zero as $|x|+|t| \rightarrow \infty$ faster than any rational function is denoted $S$ or $S\left(R^{n+1}\right)$ and is given the usual topology. See [14]. Its dual space, the space of tempered distributions on $\mathrm{R}^{n+1}$, is denoted $\mathcal{S}^{\prime}$ or $\mathcal{S}^{\prime}\left(\mathrm{R}^{n+1}\right)$, and is given the usual topology. The Fourier transform of a function $\varphi \varepsilon S$ is denoted $\hat{\varphi}$ and is defined as

$$
\hat{\varphi}(\xi, \tau)=\int \varphi(x, t) \exp \{-i x \cdot \xi-i t \tau\} d x d t .
$$

The Fourier transform is extended in the usual way to $L^{2}$ and to $s^{\prime}$. For the properties see [14]. The space of functions in $\delta$ having compact support is denoted $C_{0}^{\infty}\left(\mathbf{R}^{n+1}\right)$.

The norms of primary importance for this paper are of two types. Given a function $u(x, t ; y)$ on $\mathbf{R}_{+}^{n+2}$ and exponents $p, q \boldsymbol{\varepsilon}[1, \infty]$, set

$$
\|u\|_{p q}=L^{a}(d y / y)-\text { norm of }\|u(y)\|_{p} .
$$

That is,

$$
\begin{aligned}
& \|\left. u\right|_{\mid p q}=\left(\int_{0}^{\infty}\|u(y)\|_{p}^{a} d y / y\right)^{1 / q}, \quad 1 \leqq q<\infty, \\
& \|u\|_{p \infty}=\underset{\substack{\text { ess. } \sup \\
0<y<\infty}}{ }\|u(y)\|_{p} \text {. }
\end{aligned}
$$


Given a function $v(x, t ; z, s)$ on $\mathrm{R}^{n+1} \times \mathrm{R}^{n+1}$, set

$$
\|v\|_{p q}=L^{a}\left(\frac{d z d s}{\left(|z|+|s|^{1 / 2}\right)^{n+2}}\right)-\text { norm of }\|v(\cdot, \cdot ; z, s)\|_{p} .
$$

That is, define $V(z, s)$ to be the $L^{p}\left(\mathbf{R}^{n+1}\right)$ - norm of the function $(x, t) \rightarrow v(x, t ; z, s)$, and then define

$$
\begin{aligned}
& \|v\|_{p q}=\left[\int_{\mathbf{R}^{n+1}} V(z, s)^{q} \frac{d z d s}{\left(|z|+|s|^{1 / 2}\right)^{n+2}}\right]^{1 / q}, \quad 1 \leqq q<\infty, \\
& \|v\|_{p \infty}={\operatorname{ess.~} \sup _{(z, s) \varepsilon \mathbf{R}^{n+1}} V(z, s) .}^{\text {end }} .
\end{aligned}
$$

Various properties of these norms are contained in the appendix.

The notation $c$ will be used generically for constants appearing in certain estimates, so that $c$ need not be the same at different occurrences.

2. The heat equation in a half space. In this section we must deal with the problem of representing solutions of the heat equation in a half space in terms of boundary values of the solutions. The situation is this: $u$ satisfies the heat equation $H u=0$, where

$$
H=\frac{\partial}{\partial t}-\Delta-\frac{\partial^{2}}{\partial y^{2}}, \quad \Delta=\frac{\partial^{2}}{\partial x_{1}^{2}}+\cdots+\frac{\partial^{2}}{\partial x_{n}^{2}},
$$

in the half space $\mathbf{R}_{+}^{n+2}=\left\{(x, t, y): x \varepsilon \mathbf{R}^{n}, t \varepsilon \mathbf{R}, y>0\right\}$. Here it is possible that $n=0$, in which case $H=\partial / \partial t-\partial^{2} / \partial y^{2}$ and the form of our results must be interpreted in an obvious fashion.

The "Poisson kernel" for this problem will be seen to be the function

$$
\Gamma(x, t ; y)= \begin{cases}\frac{y}{(4 \pi)^{(n+1) / 2} t^{(n+3) / 2}} \exp \left\{-\frac{|x|^{2}+y^{2}}{4 t}\right\}, & t>0, \\ 0, & t \leqq 0,\end{cases}
$$

where $|x|$ is the Euclidean norm of the vector $x=\left(x_{1}, \cdots, x_{n}\right)$. Direct computation shows that

$$
\begin{gathered}
H \Gamma=0 \quad \text { in } \mathbf{R}_{+}^{n+2}, \\
\int_{\mathbf{R}^{n+1}} \Gamma(x, t ; y) d x d t=1, \quad y>0, \\
\lim _{y \rightarrow 0} \int_{|x|+|t| \geqq a} \Gamma(x, t ; y) d x d t=0 \text { for each } a>0 .
\end{gathered}
$$

For example, to prove (2.4) note that for $t>0$

$$
\int_{\mathbf{R}^{n}} \Gamma(x, t ; y) d x=\frac{y}{2 \pi^{1 / 2} t^{3 / 2}} \exp \left(-y^{2} / 4 t\right)
$$


and

$$
\int_{\mathbf{R}} \Gamma(x, t ; y) d t=\frac{\Gamma\left(\frac{n+1}{2}\right)}{\pi^{(n+1) / 2}} \frac{y}{\left(|x|^{2}+y^{2}\right)^{(n+1) / 2}}
$$

Thus,

$$
\begin{aligned}
\int_{|x|+|t| \geqq a} \Gamma(x, t ; y) d x d t \\
\quad \leqq \int_{a / 2}^{\infty} \frac{y}{2 \pi^{1 / 2} t^{3 / 2}} \exp \left(-y^{2} / 4 t\right) d t+\int_{|x| \geqq a / 2} \frac{\Gamma\left(\frac{n+1}{2}\right)}{\pi^{(n+1) / 2}} \frac{y}{\left(|x|^{2}+y^{2}\right)^{(n+1) / 2}} d x \\
=\int_{a / 2 y^{2}}^{\infty} \frac{1}{2 \pi^{1 / 2} \tau^{3 / 2}} \exp (-1 / 4 \tau) d \tau+\int_{|z| \geqq a / 2 y} \frac{\Gamma\left(\frac{n+1}{2}\right)}{\pi^{(n+1) / 2}} \frac{d z}{\left(|z|^{2}+1\right)^{(n+1) / 2}}
\end{aligned}
$$

and each of these integrals tends to zero as $y \rightarrow 0$.

Making the change of variable $x=y z, t=y^{2} \tau$ (as above), it is easily seen that for $1 \leqq p \leqq \infty$

$$
\|\Gamma(y)\|_{p}=A_{p, n} y^{-(n+2) / p^{\prime}}, \quad 0<y<\infty .
$$

For a function $f$ defined for $(x, t) \varepsilon \mathrm{R}^{n+1}$, define if possible

$$
(\Gamma f)(x, t ; y)=\int_{\mathbf{R}^{n+1}} \Gamma(x-z, t-s ; y) f(z, s) d z d s
$$

for $(x, t, y) \varepsilon \mathrm{R}_{+}^{n+2}$. I.e., according to the notation (1.1), $\Gamma f=\Gamma * f$. We will also use the abbreviated notation $\Gamma f(x, t ; y) \equiv(\Gamma f)(x, t ; y)$. As $(2.3)$ holds and $\Gamma \geqq 0$, it follows from Young's theorem ([18], vol. I, p. 37) that if $f \varepsilon L^{p}\left(\mathbf{R}^{n+1}\right)$, $1 \leqq p \leqq \infty$, then $\Gamma f(y) \varepsilon L^{p}\left(\mathrm{R}^{n+1}\right)$ and

$$
\|\Gamma f(y)\|_{p} \leqq\|f\|_{p} .
$$

Also (2.2) implies

$$
H(\Gamma f)=0 \text { in } \mathrm{R}_{+}^{n+2} .
$$

The following result follows from standard techniques which work for kernels $\Gamma$ which satisfy (2.3), (2.4).

Lemma 1. If $f \in L^{\infty}\left(\mathrm{R}^{n+1}\right)$ and is continuous at $\left(x_{0}, t_{0}\right)$, then

$$
\lim _{(x, t, y) \rightarrow\left(x_{0}, t_{0}, 0\right)} \Gamma f(x, t ; y)=f\left(x_{0}, t_{0}\right) .
$$

If $f$ is also uniformly continuous on $\mathrm{R}^{n+1}$, then this limit is uniform for $\left(x_{0}, t_{0}\right) \varepsilon$ $\mathbf{R}^{n+1}$. If $f \varepsilon L^{p}\left(\mathbf{R}^{n+1}\right)$ for some $p \varepsilon[1, \infty)$, then $\Gamma f(y) \rightarrow f$ in $L^{p}\left(\mathbf{R}^{n+1}\right)$.

To be able to make good use of the kernel $\Gamma$, the following uniqueness theorems are needed for the "Dirichlet problem" for $H$ in $\mathrm{R}_{+}^{n+2}$. 
Lemma 2. Let $u$ be a solution of $H u=0$ in $\mathrm{R}_{+}^{n+2}$ which is bounded and continuous in $\mathrm{R}_{+}^{\overline{n+2}}$ and satisfies $u(x, t ; 0)=0$. Then $u=0$.

Proof. Let $G$ be the domain $\left\{(x, t, y):\left|x_{i}\right|<1,-1<t<0,0<y<1\right\}$ and let $B$ be its "parabolic boundary": $B=\left\{(x, t, y):\left|x_{i}\right|=1\right.$ for some $i$ or $y=0$ or $y=1$ or $t=-1$ \}. Let $f$ be a continuous function on $B$ which satisfies

$$
\begin{aligned}
f(x, t ; 1)= & f(x,-1 ; y)=1 \\
f(x, t ; y)= & 1 \text { if }\left|x_{i}\right|=1 \text { for some } i \\
f(0,0 ; 0)= & 0 \\
& 0 \leqq f .
\end{aligned}
$$

It is well known [5] that there exists a unique solution $\omega$ of the heat equation $H \omega=0$ in $G$ which is continuous in the closure of $G$ and equals $f$ on $B$. Let $M$ be chosen such that $|u(x, t ; y)| \leqq M$ in $\mathrm{R}_{+}^{n+2}$. Let $A$ be any positive number and define $v(x, t ; y)=u\left(A x, A^{2} t ; A y\right)-M \omega(x, t ; y)$. Then $v$ is a solution of the heat equation in $G$ and the choice of $f$ shows that $v \leqq 0$ in $B$. The maximum principle [11] implies that $v \leqq 0$ in the closure of $G$. Thus, if $\left|x_{i}\right|<1$ and $0<y<1$, $u(A x, 0 ; A y) \leqq M \omega(x, 0 ; y)$. Let $x_{0} \varepsilon \mathrm{R}^{n}$ and $y_{0}>0$ be arbitrary and set $A x=x_{0}$, $A y=y_{0}$ with $A$ sufficiently large that $\left|x_{i}\right|<1,0<y<1$. Let $A \rightarrow \infty$ to obtain $u\left(x_{0}, 0 ; y_{0}\right) \leqq M \omega(0,0 ; 0)=M f(0,0 ; 0)=0$. Thus, $u \leqq 0$ for $t=0$, and translation invariance shows $u \leqq 0$ in $\mathrm{R}_{+}^{n+2}$. Likewise, $u \geqq 0$ in $\mathrm{R}_{+}^{n+2}$.

Lemma 3. Let $u$ be a solution of $H u=0$ in $\mathrm{R}_{+}^{n+2}$ which is continuous in $\mathbf{R}_{+}^{\overline{n+2}}$ and satisfies $u(x, t ; 0)=0$. Let $1 \leqq p \leqq \infty$ and assume that for some constant $M$

$$
\|u(y)\|_{p} \leqq M, \quad 0<y<\infty \text {. }
$$

Then $u=0$.

Proof. Let $\varphi \varepsilon C_{0}^{\infty}\left(\mathbf{R}^{n+1}\right)$ and set $v=\varphi * u$. Then also $H v=0$ in $\mathbf{R}_{+}^{n+2}$, and Hölder's inequality implies

$$
\|v(y)\|_{\infty} \leqq\|\varphi\|_{p^{\prime}}\|u(y)\|_{p} .
$$

Thus $v$ is bounded and continuous in $\mathrm{R}_{+}^{\overline{n+2}}$ and satisfies $v(x, t ; 0)=0$. Lemma 2 shows that $v=0$. But since $\varphi * u=0$ for every such function $\varphi$, also $u=0$.

Lemma 4. Let $u$ be a solution of $H u=0$ in $\mathbf{R}_{+}^{n+2}$ which satisfies (2.7) for some $p \varepsilon[1, \infty]$. If $y_{1}$ and $y_{2}$ are positive, then

$$
u\left(y_{1}+y_{2}\right)=\Gamma\left(y_{1}\right) * u\left(y_{2}\right)
$$

Proof. Let $v(y)=u\left(y+y_{2}\right)-\Gamma(y) * u\left(y_{2}\right)$. Then $H v=0$ in $\mathrm{R}_{+}^{n+2}$ and (2.6), (2.7) imply $\|v(y)\|_{p} \leqq 2 M$. Moreover, $v$ is continuous for $y \geqq 0$ and satisfies $v(x, t ; 0)=0$ by Lemma 1 . Thus, Lemma 3 implies $v=0$.

It is convenient at this stage to discuss the pointwise limit properties of solutions of $H u=0$. For the sake of completeness this will be carried out not only 
for solutions of the form $\Gamma f$, but also the generalization which allows $f$ to be a measure will be considered. Also a discussion of "non-tangential" limits will be given.

Definition. For $(x, t) \varepsilon \mathrm{R}^{n+1}$ and $a>0$ let $E_{a}(x, t)=\left\{(z, s) \varepsilon \mathrm{R}^{n+1}:|z-x|<a\right.$, $\left.|s-t|<a^{2}\right\}$.

Definition. For $(x, t) \varepsilon \mathrm{R}^{n+1}$ and $\alpha>0$ let

$$
P_{\alpha}(x, t)=\left\{(z, s, y) \varepsilon \mathbf{R}_{+}^{n+2}:|z-x|<\alpha y,|s-t|<\alpha^{2} y^{2}\right\} .
$$

Definition. For a non negative Borel measure $\mu$ on $\mathrm{R}^{n+1}$ define the maximal function $\mu^{*}$ to be

$$
\mu^{*}(x, t)=\sup _{a>0} a^{-(n+2)} \mu\left(B_{a}(x, t)\right)
$$

Also define

$$
\tilde{\mu}(x, t)=\limsup _{a \rightarrow 0} a^{-(n+2)} \mu\left(B_{a}(x, t)\right) .
$$

The properties of the maximal function $\mu^{*}$ relevant for our purposes can be found in [3], [7]. If $\mu$ has finite total variation on $\mathrm{R}^{n+1}$, then $\mu^{*}<\infty$ a.e. And if $f \varepsilon L_{l o c}^{1}\left(\mathbf{R}^{n+1}\right)$, then almost all points $(x, t)$ of $\mathbf{R}^{n+1}$ belong to the "modified Lebesgue set" of $f$; precisely, for almost all $(x, t)$,

$$
\lim _{a \rightarrow 0} a^{-(n+2)} \int_{B_{a}(x, t)}|f(z, s)-f(x, t)| d z d s=0 .
$$

If $\mu$ is a complex Borel measure on $\mathrm{R}^{n+1}$ with finite total variation, set

$$
\Gamma \mu(x, t ; y)=\int \Gamma(x-z, t-s ; y) d \mu(z, s) .
$$

Lemma 5. For a non negative Borel measure $\mu$ on $\mathrm{R}^{n+1}$,

$$
\sup _{P_{\alpha}(x, t)} \Gamma \mu \leqq C \mu^{*}(x, t)
$$

$$
\limsup _{\substack{(z, s, y) \varepsilon P_{\alpha}(x, t) \\ y \rightarrow 0}} \Gamma \mu(z, s ; y) \leqq C \tilde{\mu}(x, t) \quad \text { if } \quad \mu^{*}(x, t)<\infty .
$$

Here $C$ is a constant which depends only on $n$ and $\alpha$.

Proof. By translation invariance it can be assumed that $(x, t)=(0,0)$. Define $U_{k}=B_{2^{k} \alpha y}(0,0), k \geqq 1, U_{0}=\varnothing$. Then for $\left(x_{0}, t_{0}, y\right) \varepsilon P_{\alpha}(0,0)$

$$
\Gamma \mu\left(x_{0}, t_{0} ; y\right) \leqq \sum_{k=1}^{\infty} \sup _{(z, s) \varepsilon U_{k}-U_{k-1}} \Gamma\left(x_{0}-z, t_{0}-s ; y\right) \mu\left(U_{k}-U_{k-1}\right) .
$$

Now $\left|x_{0}\right|<\alpha y$ and $\left|t_{0}\right|<\alpha^{2} y^{2}$, so it follows that if $(z, s) \notin U_{k-1}$ for $k \geqq 2$, then either $\left|x_{0}-z\right|>|z|-\alpha y \geqq 2^{k-1} \alpha y-\alpha y \geqq 2^{k-2} \alpha y$ or $\left|t_{0}-s\right|>|s|-\alpha^{2} y^{2} \geqq$ 
$2^{2(k-1)} \alpha^{2} y^{2}-\alpha^{2} y^{2} \geqq 2^{2(k-2)} \alpha^{2} y^{2}$. Thus, using (2.1)

$$
\begin{aligned}
\sup _{(z, s) \notin U k-1} & \Gamma\left(x_{0}-z, t_{0}-s ; y\right) \\
& \leqq(4 \pi)^{-(n+1) / 2} \max \left\{\frac{y}{\left(t_{0}-s\right)^{(n+3) / 2}} \exp \left(-\frac{2^{2 k-4} \alpha^{2} y^{2}}{4\left(t_{0}-s\right)}\right), \frac{y}{\left(2^{2 k-4} \alpha^{2} y^{2}\right)^{(n+3) / 2}}\right\} \\
& \leqq C_{1} y^{-n-2} 2^{-k(n+3)},
\end{aligned}
$$

where $C_{1}$ depends only on $\alpha$ and $n$. The same inequality holds for $k=1$ by (2.5) for $p=\infty$. Thus, (2.11) implies

$$
\begin{aligned}
\Gamma \mu\left(x_{0}, t_{0} ; y\right) & \leqq C_{1} \sum_{k=1}^{\infty} 2^{-k(n+3)} \frac{\mu\left(U_{k}\right)}{\left(2^{k} \alpha y\right)^{n+2}} 2^{k(n+2)} \alpha^{n+2} \\
& =C \sum_{k=1}^{\infty} 2^{-k} \frac{\mu\left(U_{k}\right)}{\left(2^{k} \alpha y\right)^{n+2}} .
\end{aligned}
$$

This implies immediately that $\Gamma \mu\left(x_{0}, t_{0} ; y\right) \leqq C \mu^{*}(0,0)$, proving (2.9). Since for each $k$

$$
\limsup _{y \rightarrow 0} \frac{\mu\left(U_{k}\right)}{\left(2^{k} \alpha y\right)^{n+2}}=\tilde{\mu}(0,0),
$$

(2.10) also follows from (2.12).

The basic result on pointwise limits in this setting can now be given.

Theorem 1. Let $f \varepsilon L^{p}\left(\mathrm{R}^{n+1}\right)$. Then for almost all points $(x, t) \varepsilon \mathrm{R}^{n+1}$

$$
\lim _{(z, s, y) \varepsilon P_{\alpha}(x, t)} \Gamma f(z, s ; y)=f(x, t) .
$$

Let $\mu$ be a complex Borel measure on $\mathbf{R}^{n+1}$ with finite total variation. Then for almost all points $(x, t) \varepsilon \mathrm{R}^{n+1}$.

$$
\lim _{\substack{(z, s, y) \varepsilon P_{\alpha}(x, t) \\ y \rightarrow 0}} \Gamma \mu(z, s ; y)=\mu^{\prime}(x, t)
$$

where

$$
\mu^{\prime}(x, t)=\lim _{a \rightarrow 0} \frac{\mu\left(B_{a}(x, t)\right)}{\lambda\left(B_{a}(x, t)\right)}
$$

where $\lambda$ is Lebesgue measure in $\mathrm{R}^{n+1}$.

Proof. According to (2.8) there exists a set $N$ of zero measure in $\mathrm{R}^{n+1}$ such that if $(x, t) \notin N$,

$$
\lim _{a \rightarrow 0} \frac{1}{a^{n+2}} \int_{B_{a}(x, t)}|f(z, s)-f(x, t)| d z d s=0 .
$$


Assume $(x, t) \notin N$ and write for $(z, s, y) \varepsilon P_{\alpha}(x, t)$

$$
\begin{aligned}
|\Gamma f(z, s ; y)-f(x, t)| & =\left|\int \Gamma\left(z-z^{\prime}, s-s^{\prime} ; y\right)\left[f\left(z^{\prime}, s^{\prime}\right)-f(x, t)\right] d z^{\prime} d s^{\prime}\right| \\
& \leqq \int \Gamma\left(z-z^{\prime}, s-s^{\prime} ; y\right) d \sigma\left(z^{\prime}, s^{\prime}\right),
\end{aligned}
$$

where $\sigma$ is the Borel measure on $\mathrm{R}^{n+1}$ defined by

$$
\sigma(E)=\int_{E}\left|f\left(z^{\prime}, s^{\prime}\right)-f(x, t)\right| d z^{\prime} d s^{\prime}
$$

for any Borel set $E$. Now (2.15) shows that $\tilde{\sigma}(x, t)=0$. And since Hölder's inequality implies $\sigma(E) \leqq\|f\|_{p} \lambda(E)^{1 / \nu^{\prime}}+|f(x, t)| \lambda(E)$, it follows that $\sigma^{*}(x, t)$ $<\infty$. Thus, Lemma 5 shows that

$$
\lim _{\substack{(z, s, y) \mathcal{P}_{\alpha} \alpha(x, t) \\ y \rightarrow 0}}|\Gamma f(z, s ; y)-f(x, t)| \leqq C \tilde{\sigma}(x, t)=0
$$

proving (2.13). To prove (2.14) write the Lebesgue decomposition of $\mu$ in the form $\mu=g \lambda+\nu$, where $g \varepsilon L^{1}\left(\mathbf{R}^{n+1}\right)$ and $\nu$ is singular with respect to $\lambda$. Again [3] shows that if $|\nu|$ is the total variation of $\nu$, then $|\nu|^{\prime}(x, t)=0$ almost everywhere. Using (2.13) to obtain the correct limit for $\Gamma g$ almost everywhere, it follows that (2.14) will be proved if it is shown that $\Gamma \nu$ tends to 0 almost everywhere. But if $|\nu|^{\prime}(x, t)=0$, then Lemma 5 shows

$$
\begin{aligned}
\limsup _{\substack{(z, s, y) \operatorname{se}_{\alpha}(x, t) \\
\nu \rightarrow 0}}|\Gamma \nu(z, s ; y)| & \leqq \lim _{\substack{(z, s, y) \sup _{\alpha}(x, t) \\
y \rightarrow 0}} \Gamma|\nu|(z, s ; y) \\
& \leqq C \widetilde{|\nu|}(x, t) \\
& =C|\nu|^{\prime}(x, t)=0 .
\end{aligned}
$$

Lemma 6. Let $u$ be a solution of $H u=0$ in $\mathrm{R}_{+}^{n+2}$ which satisfies

$$
\|u(y)\|_{p} \leqq M, \quad 0<y<\infty,
$$

for some $p \varepsilon(1, \infty]$. Then there exists a unique $f \varepsilon L^{p}\left(\mathbf{R}^{n+1}\right)$ such that $u=\Gamma f$. Moreover, $\|f\|_{p} \leqq M$.

Proof. The uniqueness of $f$ follows from Theorem 1. As $L^{p}$ is the dual space of $L^{p^{\prime}}$, the assumptions show that there exists $f \varepsilon L^{p}$ which is a weak accumulation point of the elements $u\left(k^{-1}\right)$ of $L^{p}$. As $\Gamma(x-\cdot, t-\cdot ; y) \varepsilon L^{p^{\prime}}\left(R^{n+1}\right)$ by (2.5), it follows that for some sequence $k_{i} \rightarrow \infty$

$$
\begin{aligned}
\int \Gamma(x-z, t-s ; y) f(z, s) d z d s & =\lim _{i \rightarrow \infty} \int \Gamma(x-z, t-s ; y) u\left(z, s ; k_{j}^{-1}\right) d z d s \\
& =\lim _{i \rightarrow \infty} u\left(x, t ; y+k_{i}^{-1}\right) \quad \text { (Lemma 4) } \\
& =u(x, t ; y) .
\end{aligned}
$$


That is, $\Gamma f=u$.

Lemma 7. Let $\mu$ be a totally finite complex Borel measure on $\mathrm{R}^{n+1}$. Then

$$
\lim _{y \rightarrow 0} \Gamma \mu(y)=\mu
$$

in the sense of weak convergence of measures.

Proof. Let $\varphi$ be a continuous function with compact support in $\mathrm{R}^{n+1}$. Then Fubini's theorem gives

$$
\int \Gamma \mu(x, t ; y) \varphi(x, t) d x d t=\int d \mu(z, s) \int \Gamma(x-z, t-s ; y) \varphi(x, t) d x d t
$$

Lemma 1 shows that the function

$\int \Gamma(x-z, t-s ; y) \varphi(x, t) d x d t=\int \Gamma(-z-x,-s-t ; y) \varphi(-x,-t) d x d t$ converges uniformly to $\varphi(z, s)$ as $y \rightarrow 0$. Thus,

$$
\lim _{y \rightarrow 0} \int \Gamma \mu(x, t ; y) \varphi(x, t) d x d t=\int \varphi(z, s) d \mu(z, s) .
$$

Now a result for $p=1$ corresponding to Lemma 6 can be given.

Lemma 8. Let $u$ be a solution of $H u=0$ in $\mathrm{R}_{+}^{n+2}$ which satisfies

$$
\|u(y)\|_{1} \leqq M, 0<y<\infty .
$$

Then there exists a unique complex Borel measure $\mu$ on $\mathrm{R}^{n+1}$ such that $u=\Gamma \mu$. Moreover, $|\mu|\left(\mathrm{R}^{n+1}\right) \leqq M$.

Proof. Uniqueness follows from Lemma 7. As the space of bounded measures on $\mathrm{R}^{n+1}$ is the dual space of the Banach space of bounded continuous functions on $\mathrm{R}^{n+1}$ which vanish at infinity it follows that there exists a measure $\mu$ which is a weak accumulation point of the absolutely continuous measures $u\left(k^{-1}\right) d \lambda$. The proof now follows as in Lemma 6.

As a preliminary to the following succession of results, an estimate is needed for derivatives of the Poisson kernel $\Gamma$. This can be given in the following form. For any multi-index $\alpha=\left(\alpha_{1}, \ldots, \alpha_{n}\right)$ and any non negative integers $a$ and $b$, and $t>0$,

$$
\left|D_{x}^{\alpha} D_{y}^{a} D_{t}^{b} \Gamma(x, t ; y)\right| \leqq C t^{-(n+|\alpha|+a+2 b+2) / 2} \exp \left\{-\left(|x|^{2}+y^{2}\right) / 8 t\right\} .
$$

A similar estimation shows that

$$
\left|D_{x}^{\alpha} D_{y}^{a} D_{t}^{b} \Gamma(x, t ; y)\right| \leqq C y^{-|\alpha|-a-2 b} \Gamma(x, 2 t ; y) .
$$

The constant $C$ depends only on $\alpha, a, b$, and $n$. This can be easily checked, so the proof will be omitted.

Now we turn to a different class of properties of solutions of $H u=0$. First, 
define $\mathcal{F}$ to be the class of solutions $u$ of $H u=0$ in $\mathrm{R}_{+}^{n+2}$ such that $u$ is bounded in every half space $y \geqq \epsilon>0$, and let $\mathcal{F C}_{0}$ be the class of functions $u$ which belong to $\mathfrak{F}$ and also satisfy the condition $\lim _{y \rightarrow \infty} u(x, t ; y)=0$ for all $(x, t) \varepsilon \mathbf{R}^{n+1}$.

Now if $u \varepsilon \mathfrak{F C}$, then Lemma 4 applied to the half space $y \geqq \epsilon$ shows that for all $y_{1}>0, y_{2}>0$,

$$
u\left(y_{1}+y_{2}\right)=\Gamma\left(y_{1}\right) * u\left(y_{2}\right) .
$$

If (2.18) is differentiated with respect to $x_{i}, t, y_{1}$, or $y_{2}$, the following relations are obtained:

$$
\begin{aligned}
& u_{x_{i}}\left(y_{1}+y_{2}\right)=\Gamma_{x_{i}}\left(y_{1}\right) * u\left(y_{2}\right)=\Gamma\left(y_{1}\right) * u_{x_{i}}\left(y_{2}\right) \\
& u_{t}\left(y_{1}+y_{2}\right)=\Gamma_{t}\left(y_{1}\right) * u\left(y_{2}\right)=\Gamma\left(y_{1}\right) * u_{t}\left(y_{2}\right) \\
& u_{y}\left(y_{1}+y_{2}\right)=\Gamma_{y}\left(y_{1}\right) * u\left(y_{2}\right)=\Gamma\left(y_{1}\right) * u_{y}\left(y_{2}\right)
\end{aligned}
$$

Applying Young's inequality and (2.17) to these relations gives for $1 \leqq p \leqq \infty$

$$
\begin{aligned}
\left\|u_{x_{i}}(y)\right\|_{p} & \leqq C y^{-1}\|u(y / 2)\|_{p} \\
\left\|u_{t}(y)\right\|_{p} & \leqq C y^{-2}\|u(y / 2)\|_{p} \\
\left\|u_{y}(y)\right\|_{p} & \leqq C y^{-1}\|u(y / 2)\|_{p} .
\end{aligned}
$$

These estimates imply immediately the following estimates for the norms \|\|$_{p e}$ as defined in the introduction.

Lemma 9. Let $u \varepsilon \mathfrak{F}$ and $\beta \varepsilon \mathrm{R}$. Then

$$
\begin{aligned}
\left\|y^{\beta+1} u_{x_{i}}\right\|_{p q} & \leqq C 2^{\beta}\left\|y^{\beta} u\right\|_{p q} ; \\
\left\|y^{\beta+2} u_{t}\right\|_{p q} & \leqq C 2^{\beta}\left\|y^{\beta} u\right\|_{p q} ; \\
\left\|y^{\beta+1} u_{y}\right\|_{p q} & \leqq C 2^{\beta}\left\|y^{\beta} u\right\|_{p q}
\end{aligned}
$$

The converse of these estimates depends on the following result, which in reality has nothing to do with the heat equation, but is only a version of Hardy's lemma.

Lemma 10. Let $u \in \mathfrak{H}_{0}$ and $\beta>0$. Then

$$
\left\|y^{\beta} u\right\|_{p q} \leqq \beta^{-1}\left\|y^{\beta+1} u_{y}\right\|_{\boldsymbol{D} \text {. }}
$$

Proof. By definition of $\mathfrak{H}_{0}$

$$
|u(x, t ; y)| \leqq \int_{y}^{\infty}\left|u_{\eta}(x, t ; \eta)\right| d \eta
$$

Thus it follows that

$$
\|u(y)\|_{p} \leqq \int_{y}^{\infty}\left\|u_{\eta}(\eta)\right\|_{p} d \eta
$$

Then Lemma $E$ of the appendix applies directly to give the result. 
Theorem 2. Let $u \varepsilon$ JC and $\beta>0$. Then

$$
\begin{gathered}
C^{-1}\left\|y^{\beta} u_{y}\right\|_{p q} \leqq \sum_{i=1}^{n}\left\|y^{\beta} u_{x_{i}}\right\|_{p q}+\left\|y^{\beta+1} u_{t}\right\|_{p q} \leqq C\left\|y^{\beta} u_{y}\right\|_{p q} \\
C^{-1}\left\|y^{\beta} u_{y y}\right\|_{p q} \leqq \sum_{i, j=1}^{n}\left\|y^{\beta} u_{x_{i x}}\right\|_{p q}+\left\|y^{\beta} u_{t}\right\|_{p q} \leqq C\left\|y^{\beta} u_{y y}\right\|_{p q}
\end{gathered}
$$

Here $C$ is a constant which depends only on $\beta$ and $n$.

Proof. Note that (2.19)-(2.21) and the estimates for derivatives of $\Gamma$ in (2.17) show that if $u \in \mathfrak{F}$, then any derivative of $u$ is in $\mathfrak{H}_{0}$. Thus, we have from Lemma 9 applied to $u_{y}$ that

$$
\left\|y^{\beta+1} u_{x_{i} y}\right\|_{p q}+\left\|y^{\beta+2} u_{t y}\right\|_{p q} \leqq C_{1}\left\|y^{\beta} u_{y}\right\|_{p q} .
$$

From Lemma 10 applied to $u_{x_{i}}$ and $u_{t}$,

$$
\begin{aligned}
\left\|y^{\beta} u_{x_{i}}\right\|_{p q}+\left\|y^{\beta+1} u_{t}\right\|_{p q} & \leqq C_{2}\left\|y^{\beta+1} u_{x_{i y}}\right\|_{p q}+C_{2}\left\|y^{\beta+2} u_{t y}\right\|_{p q} \\
& \leqq C_{1} C_{2}\left\|y^{\beta} u_{y}\right\|_{p q} .
\end{aligned}
$$

This establishes the right inequality of (2.23). Applying Lemma 10 to $u_{y}$ and using $H u=0$,

$$
\begin{aligned}
\left\|y^{\beta} u_{y}\right\|_{p q} & \leqq \beta^{-1}\left\|y^{\beta+1} u_{y y}\right\|_{p q} \\
& =\beta^{-1}\left\|y^{\beta+1} u_{t}-y^{\beta+1} \Delta u\right\|_{p q} \\
& \leqq \beta^{-1}\left\|y^{\beta+1} u_{t}\right\|_{p q}+\sum_{i=1}^{n} \beta^{-1}\left\|y^{\beta+1} u_{x_{i} x_{i}}\right\|_{p q} \\
& \leqq \beta^{-1}\left\|y^{\beta+1} u_{t}\right\|_{p q}+\sum_{i=1}^{n} \beta^{-1} C_{1}\left\|y^{\beta} u_{x_{i}}\right\|_{p q}
\end{aligned}
$$

where Lemma 9 was used at the last step. Thus, (2.23) is proved. The left inequality of (2.24) is a trivial consequence of the relation $H u=0$. The right inequality follows from (2.23):

$$
\left\|\left.y^{\beta} u_{x_{i} x_{j}}\right|_{p q} \leqq\left. C_{3}|| y^{\beta} u_{x_{i y}}\right|_{\left.\right|_{p q}} \leqq C_{3}^{2}\right\| y^{\beta} u_{y y} \|_{p q}
$$

and the relation $H u=0$.

One more simple consequence of Lemmas 9 and 10 will be mentioned.

Lemma 11. Let $u \varepsilon \mathfrak{F C}, \beta>0$, and $k$ be a positive integer. Then there exists a constant $C=C(\beta, k, n)$ such that

$$
C^{-1}\left\|y^{\beta} D_{y} u\right\|_{p q} \leqq\left\|y^{\beta+k-1} D_{y}^{k} u\right\|_{p q} \leqq C\left\|y^{\beta} D_{y} u\right\|_{p q} \text {. }
$$

Remark. If $u$ is a solution of $H u=0$ in $\mathrm{R}_{+}^{n+2}$ and satisfies the condition $\sup _{y>0}\|u(y)\|_{p}<\infty$, then $u \varepsilon \mathfrak{H}$. For Lemma 4, Hölder's inequality, and (2.5) show that

$$
|u(x, t ; y)| \leqq \text { const } y^{-(n+2) / p} .
$$


In particular, if $f \varepsilon L^{p}\left(\mathrm{R}^{n+1}\right)$, then $\Gamma f \varepsilon \mathfrak{F}$.

Although it will not be needed in this paper, the formula

$$
\hat{\Gamma}(\xi, \tau ; y)=\exp \left\{-\left(|\xi|^{2}+i \tau\right)^{1 / 2} y\right\}
$$

will now be derived, since it is needed in [9]. Of course, this could be computed directly, but a simpler procedure is available. It follows upon taking the Fourier transform of the equation $H \Gamma=0$ that

$$
i \tau \hat{\Gamma}(y)+|\xi|^{2} \hat{\Gamma}(y)-\frac{\partial^{2}}{\partial y^{2}} \hat{\Gamma}(y)=0 .
$$

A basis for solutions of this ordinary differential equation is the pair of functions $\exp \left\{ \pm\left(|\xi|^{2}+i \tau\right)^{1 / 2} y\right\}$, where the principal determination of the square root is indicated. Since $\Gamma(y) \rightarrow 0$ as $y \rightarrow \infty$ in the topology of $L^{2}\left(\mathbf{R}^{n+1}\right)(c f .(2.5))$, it follows that

$$
\hat{\Gamma}(\xi, \tau ; y)=a(\xi, \tau) \exp \left\{-\left(|\xi|^{2}+i \tau\right)^{1 / 2} y\right\} .
$$

Since as $y \rightarrow 0, \Gamma(y) \rightarrow \delta$ (the Dirac measure at the origin) in the topology of $\boldsymbol{s}^{\prime}$, it follows that $\hat{\Gamma}(y) \rightarrow 1$ in the same topology. Thus, $a(\xi, \tau) \equiv 1$.

3. Lipschitz spaces. The preliminaries of the preceding section now allow a discussion of the boundary behavior of $\Gamma f$ as it relates to the properties of $f$. First, in analogy with the spaces $\Lambda(\alpha ; p, q)$ of [15] the following definition is made.

Definition. Let $p, q \varepsilon[1, \infty]$ and let $\alpha>0$. Let $k>\alpha$ be an integer. Then $\Lambda^{\prime}(\alpha ; p, q)$ is the subspace of $L^{p}\left(\mathbf{R}^{n+1}\right)$ consisting of those $f$ which satisfy $\left\|y^{k-\alpha} D_{v}^{k} \Gamma f\right\|_{p q}<\infty$.

Lemma 11 and the remark following it show easily that $\Lambda^{\prime}(\alpha ; p, q)$ is independent of $k$. This space can be supplied with the norm

$$
\|f\|_{\alpha ; p, \alpha}=\|f\|_{L^{p}\left(\mathrm{R}^{n+1}\right)}+\left\|y^{k-\alpha} D_{y}^{k} \Gamma f\right\|_{p q}
$$

and the norms for various $k>\alpha$ are mutually equivalent. Thus, $\Lambda^{\prime}(\alpha ; p, q)$ is a normable space, and it is moreover easily checked that $\Lambda^{\prime}(\alpha ; p, q)$ is complete.

Now the problem arises of the possibility of giving explicitly in terms of properties $f$ alone (without using the Poisson integral $\Gamma f$ ) some necessary and sufficient conditions that $f \varepsilon \Lambda^{\prime}(\alpha ; p, q)$. A partial solution to this problem will now be discussed, using arguments similar to those in Theorem 4 of [15].

For $(z, s) \varepsilon \mathrm{R}^{n+1}$ define $\left(\tau_{z, s} f\right)(x, t)=f(x-z, t-s)$.

Theorem 3. Let $p, q \varepsilon[1, \infty]$ and for $f \varepsilon L^{p}\left(\mathrm{R}^{n+1}\right)$ let

$$
\begin{aligned}
A & =\left\|\frac{\tau_{z, s} f-f}{\left(|z|+|s|^{1 / 2}\right)^{\alpha}}\right\|_{p q}, \\
B_{1} & =L^{\alpha}\left(\frac{d z}{|z|^{n}}\right)-\text { norm of the function } z \rightarrow \frac{\left\|\tau_{z, 0} f-2 f+\tau_{-z, 0} f\right\|_{p}}{|z|^{\alpha}},
\end{aligned}
$$




$$
\begin{aligned}
B_{2} & =L^{a}\left(\frac{d s}{|s|}\right)-\text { norm of the function } s \rightarrow \frac{\left\|\tau_{0, s} f-f\right\|_{p}}{|s|^{\alpha / 2}}, \\
B & =B_{1}+B_{2} \\
C & =\left\|y^{2-\alpha} D_{y}^{2} \Gamma f\right\|_{p q}, \\
D & =\left\|y^{-\alpha}(\Gamma f(y)-f)\right\|_{p q}, \\
E & =\left\|y^{-\alpha}(\Gamma f(2 y)-2 \Gamma f(y)+f)\right\|_{p q} .
\end{aligned}
$$

If $0<\alpha<1$, all the semi-norms $A-E$ are equivalent. If $0<\alpha<2$, the semi-norms $B, C, E$ are equivalent.

Proof. The equivalences relating the semi-norms $C, D$, and $E$ are really consequences of general facts about semi groups, here specialized to the semi group of operators $f \rightarrow \Gamma f(y)$; see [12]. Thus, it will be assumed known that $C, D$, and $E$ are equivalent for $0<\alpha<1$ and that $C$ and $E$ are equivalent for $0<\alpha<2$. The proof of the other results requires the special nature of the semi group and, more particularly, involves the differential operator $H$ rather strongly.

Assume $0<\alpha<1$. Then

$$
\begin{aligned}
D_{y} \Gamma f(x, t ; y) & =\int D_{\nu} \Gamma(z, s ; y) f(x-z, t-s) d z d s \\
& =\int D_{y} \Gamma(z, s ; y)[f(x-z, t-s)-f(x, t)] d z d s .
\end{aligned}
$$

Thus, Minkowski's integral inequality shows

$$
y^{1-\alpha}\left\|D_{\nu} \Gamma f(y)\right\|_{p} \leqq \int K(z, s ; y) \frac{\left\|\tau_{z, s} f-f\right\|_{p}}{\left(|z|+|s|^{1 / 2}\right)^{\alpha}} d z d s
$$

where

$$
K(z, s ; y)=y^{1-\alpha}\left(|z|+|s|^{1 / 2}\right)^{\alpha}\left|D_{y} \Gamma(z, s, y)\right| .
$$

In order to apply Lemma $\mathrm{C}$ of the appendix, it is necessary to prove the estimates

$$
\begin{array}{r}
\int_{\mathrm{R}^{n+1}} K(z, s ; y) d z d s \leqq \text { const } \\
\int_{0}^{\infty}\left(|z|+|s|^{1 / 2}\right)^{n+2} K(z, s ; y) \frac{d y}{y} \leqq \text { const. }
\end{array}
$$

Now using (2.16) the first of these two integrals is dominated by a constant multiple of

$$
\begin{aligned}
\int_{\mathbf{R}^{n+1}} y^{1-\alpha}\left(|z|+|s|^{1 / 2}\right)^{\alpha} & |s|^{-(n+3) / 2} \exp \left\{-\left(|z|^{2}+y^{2}\right) / 8|s|\right\} d z d s \\
& \leqq c \int_{\mathbf{R}^{n+1}} y^{1-\alpha}|s|^{(\alpha-n-3) / 2} \exp \left\{-\left(|z|^{2}+y^{2}\right) / 9|s|\right\} d z d s
\end{aligned}
$$




$$
\begin{aligned}
& =c \int_{0}^{\infty} y^{1-\alpha} s^{(\alpha-3) / 2} \exp \left(-y^{2} / 9 s\right) d \\
& =c \int_{0}^{\infty} s^{(\alpha-3) / 2} \exp (-1 / 9 s) d s<\infty,
\end{aligned}
$$

and the second by a constant multiple of

$$
\begin{aligned}
\int_{0}^{\infty}\left(|z|+|s|^{1 / 2}\right)^{n+2+\alpha} y^{-\alpha}|s|^{-(n+3) / 2} & \exp \left\{-\left(|z|^{2}+y^{2}\right) / 8|s|\right\} d y \\
& \leqq c \int_{0}^{\infty}|s|^{(\alpha-1) / 2} y^{-\alpha} \exp \left\{-\left(|z|^{2}+y^{2}\right) / 9||\right\} \\
& \leqq c \int_{0}^{\infty} y^{-\alpha} \exp \left(-y^{2} / 9\right) d y<\infty
\end{aligned}
$$

Thus Lemma $\mathrm{C}$ shows that $C$ is dominated by $A$.

Now to dominate $A$, write

$$
\begin{aligned}
f(x-z, t-s)-f(x, t)= & {[f(x-z, t-s)-\Gamma f(x-z, t-s ; y)] } \\
& +[\Gamma f(x, t ; y)-f(x, t)] \\
& +[\Gamma f(x-z, t-s ; y)-\Gamma f(x, t ; y)] .
\end{aligned}
$$

Then the mean value theorem shows that

$$
\begin{aligned}
& \left\|\tau_{z, s} f-f\right\|_{p} \leqq\|f-\Gamma f(y)\|_{p}+\|\Gamma f(y)-f\|_{p} \\
& \quad+|z| \sum_{i=1}^{n}\left\|D_{x_{i}} \Gamma f(y)\right\|_{p}+|s|\left\|D_{t} \Gamma f(y)\right\|_{p} .
\end{aligned}
$$

Now choose $y=|z|+|s|^{1 / 2}$. Then

$\frac{\left\|\tau_{z, s} f-f\right\|_{p}}{\left(|z|+|s|^{1 / 2}\right)^{\alpha}} \leqq 2 y^{-\alpha}\|\Gamma f(y)-f\|_{p}+\sum_{i=1}^{n} y^{1-\alpha}\left\|D_{x_{i}} \Gamma f(y)\right\|_{p}+y^{2-\alpha}\left\|D_{t} \Gamma f(y)\right\|_{p}$.

Taking the

$$
L^{Q}\left(\frac{d z d s}{\left(|z|+|s|^{1 / 2}\right)^{n+2}}\right)-\text { norm }
$$

of each side gives an estimate for $A$ in terms of norms of functions which depend only on $y=|z|+|s|^{1 / 2}$. According to Lemma $\mathrm{B}$ of the appendix, this gives

$$
\begin{aligned}
A & \leqq c\left\|y^{-\alpha}(\Gamma f(y)-f)\right\|_{p q}+c \sum_{i=1}^{n}\left\|y^{1-\alpha} D_{x_{i}} \Gamma f\right\|_{p q}+c\left\|y^{2-\alpha} D_{t} \Gamma f\right\|_{p q} \\
& \leqq c D+c\left\|y^{1-\alpha} D_{y} \Gamma f\right\|_{p q}
\end{aligned}
$$

the last inequality following from (2.23). Thus, $A$ is dominated in terms of $D$ and $C$ (cf. Lemma 11). 
Now assume $0<\alpha<2$. It must be shown that $B$ and $C$ are equivalent. Proceeding as in (3.2),

$$
\begin{aligned}
f(x-z, t)-2 f(x, t) & +f(x+z, t)=[f(x-z, t)-\Gamma f(x-z, t ; y)] \\
& -2[f(x, t)-\Gamma f(x, t ; y)]+[f(x+z, t)-\Gamma f(x+z, t ; y)] \\
& +[\Gamma f(x-z, t ; y)-2 \Gamma f(x, t ; y)+\Gamma f(x+z, t ; y)] .
\end{aligned}
$$

Note that Taylor's theorem shows $f(x, t)-\Gamma f(x, t ; y)=-y D_{y} \Gamma f(x, t ; y)+$ $\int_{0}^{y} \eta D_{\eta}^{2} \Gamma f(x, t ; \eta) d \eta$. This relation and Lemma $\mathrm{D}$ of the appendix show

$$
\begin{aligned}
\| \tau_{z, 0} f-2 f & +\tau_{-z, 0} f\left\|_{p} \leqq \sum_{i, j=1}^{n}\left|z_{i}\right|\left|z_{j}\right|\right\| D_{x_{i}} D_{x_{i}} y D_{\nu} \Gamma f(y) \|_{p} \\
& +4 \int_{0}^{y} \eta\left\|D_{\eta}^{2} \Gamma f(\eta)\right\|_{p} d \eta+\sum_{i, j=1}^{n}\left|z_{i}\right|\left|z_{j}\right|\left\|D_{x_{i}} D_{x_{i}} \Gamma f(y)\right\|_{p} .
\end{aligned}
$$

(In actual fact, this estimate should be obtained for $\Gamma f(\epsilon)$ rather than $f$ itself; then if we let $\epsilon \rightarrow 0$ and use from Theorem 1 the fact that $\Gamma f(\epsilon) \rightarrow f$ a.e., (3.3) does indeed follow.) Now set $y=|z|$ in (3.3) to obtain

$$
\begin{aligned}
& \frac{\left\|\tau_{z, 0} f-2 f+\tau_{-z, 0} f\right\|_{p}}{|z|^{\alpha}} \leqq \sum_{i, j=1}^{n} y^{3-\alpha}\left\|D_{x_{i}} D_{x_{i}} D_{y} \Gamma f(y)\right\|_{p} \\
& \quad+\sum_{i, i=1}^{n} y^{2-\alpha}\left\|D_{x_{i}} D_{x_{i}} \Gamma f(y)\right\|_{p}+4 y^{-\alpha} \int_{0}^{y} \eta\left\|D_{\eta}^{2} \Gamma f(\eta)\right\|_{p} d \eta .
\end{aligned}
$$

Taking the $L^{q}\left(d z /|z|^{n}\right)$ - norm of each side gives an estimate for $B_{1}$ in terms of norms of functions which depend only on $|z|$. According to Lemma $B$ of the appendix, the norms of the terms on the right side of (3.4) are equivalent to the $L^{q}(d y / y)$ - norms of the corresponding functions of the single variable $y$. Then applying Lemma $\mathrm{E}$ it follows that

$$
B_{1} \leqq c\left\|y^{3-\alpha} D_{x_{i x i y}} \Gamma f\right\|_{p q}+c\left\|y^{2-\alpha} D_{x_{i x i}} \Gamma f\right\|_{p q}+c\left\|y^{2-\alpha} D_{\nu}^{2} \Gamma f\right\|_{p q},
$$

and since $\alpha<2$ Theorem 2 and Lemma 11 combine to show $B_{1} \leqq c C$. An entirely similar proof gives the inequality $B_{2} \leqq c C$. The procedure is the same, with the choice $y=|s|^{1 / 2}$.

Finally, $C$ must be estimated in terms of $B$. As in (3.1),

$$
\begin{aligned}
D_{y}^{2} \Gamma f(x, t ; y) & =\int D_{\nu}^{2} \Gamma(z, s ; y)[f(x-z, t-s)-f(x, t)] d z d s \\
& =\int D_{\nu}^{2} \Gamma(z, s ; y)[f(x+z, t-s)-f(x, t)] d z d s .
\end{aligned}
$$

The last equality follows because $\Gamma(-z, s ; y)=\Gamma(z, s ; y)$. Averaging the expressions in (3.5) gives

$$
D_{\nu}^{2} \Gamma f(x, t ; y)=\int D_{u}^{2} \Gamma(z, s ; y)
$$




$$
\cdot\left[\frac{f(x-z, t-s)+f(x+z, t-s)-2 f(x, t-s)}{2}+f(x, t-s)-f(x, t)\right] d z d s .
$$

Thus, Minkowski's integral inequality shows

$$
\begin{aligned}
y^{2-\alpha}\left\|D_{y}^{2} \Gamma f(y)\right\|_{p} & \leqq \int K_{1}(z, s ; y) \frac{\left\|\tau_{z, 0} f-2 f+\tau_{-z, 0} f\right\|_{p}}{|z|^{\alpha}} d z d s \\
& +\int K_{2}(z, s ; y) \frac{\left\|\tau_{0, s} f-f\right\|_{p}}{|s|^{\alpha / 2}} d z d s,
\end{aligned}
$$

where

$$
\begin{aligned}
& K_{1}(z, s ; y)=2^{-1} y^{2-\alpha}|z|^{\alpha}\left|D_{y}^{2} \Gamma(z, s ; y)\right|, \\
& K_{2}(z, s ; y)=y^{2-\alpha}|s|^{\alpha / 2}\left|D_{y}^{2} \Gamma(z, s ; y)\right| .
\end{aligned}
$$

In order to apply Lemma $\mathrm{C}$ of the appendix it is necessary to show for $i=1,2$,

$$
\begin{array}{r}
\int_{\mathrm{R}^{n+2}} K_{i}(z, s ; y) d z d s \leqq c, \\
\int_{0}^{\infty} K_{i}(z, s ; y) \frac{\left(|z|+|s|^{1 / 2}\right)^{n+2}}{y} d y \leqq c .
\end{array}
$$

The integrals (3.7) are dominated by (cf. (2.16))

$$
\begin{aligned}
c \int_{\mathrm{R}^{n+1}} y^{2-\alpha}\left(|z|+|s|^{1 / 2}\right)^{\alpha} & |s|^{-(n+4) / 2} \exp \left\{-\left(|z|^{2}+y^{2}\right) / 8|s|\right\} d z d s \\
& \leqq c \int_{\mathrm{R}^{n+1}} y^{2-\alpha}|s|^{(\alpha-n-4) / 2} \exp \left\{-\left(|z|^{2}+y^{2}\right) / 9|s|\right\} d z d s \\
& =c \int_{0}^{\infty} y^{2-\alpha} s^{(\alpha-4) / 2} \exp \left(-y^{2} / 9 s\right) d s=c .
\end{aligned}
$$

And the integrals (3.8) are dominated by

$$
\begin{aligned}
\int_{0}^{\infty}\left(|z|+|s|^{1 / 2}\right)^{n+2+\alpha} y^{1-\alpha}|s|^{-(n+4) / 2} & \exp \left\{-\left(|z|^{2}+y^{2}\right) / 8|s|\right\} d y \\
& \leqq c \int_{0}^{\infty}|s|^{(\alpha-2) / 2} y^{1-\alpha} \exp \left(-y^{2} / 8|s|\right) d y=c .
\end{aligned}
$$

Thus Lemma C implies that the $L_{q}(d y / y)$ - norm of the left side of (3.6) is dominated by the

$$
L^{a}\left(\frac{d z d s}{\left(|z|+|s|^{1 / 2}\right)^{n+2}}\right)-\text { norm }
$$

of the expression

$$
\frac{\left\|\tau_{z, 0} f-2 f+\tau_{-z, 0} f\right\|_{p}}{|z|^{\alpha}}+\frac{\left\|\tau_{0,8} f-f\right\|_{p}}{|s|^{\alpha / 2}} .
$$


But Lemma $\mathrm{A}$ of the appendix shows that this result can be written as the inequality $C \leqq$ const $\left(B_{1}+B_{2}\right)$, proving the desired estimate for $C$ in terms of $B$.

Several more lemmas will be needed in the next section.

Lemma 12. Let $\alpha_{1}, \alpha_{2} \varepsilon(0, \infty), p, p_{1}, p_{2}, q, q_{1}, q_{2} \varepsilon[1, \infty]$, where

$$
\begin{aligned}
& \frac{1}{p}=\frac{1}{p_{1}}+\frac{1}{p_{2}}-1, \\
& \frac{1}{q}=\frac{1}{q_{1}}+\frac{1}{q_{2}} .
\end{aligned}
$$

Then

$$
\Lambda^{\prime}\left(\alpha_{1} ; p_{1}, q_{1}\right) * \Lambda^{\prime}\left(\alpha_{2} ; p_{2}, q_{2}\right) \subset \Lambda^{\prime}\left(\alpha_{1}+\alpha_{2} ; p, q\right),
$$

and the inclusion is continuous.

Proof. Let $f_{i} \varepsilon \Lambda^{\prime}\left(\alpha_{i} ; p_{i}, q_{i}\right), i=1,2$. Let $k$ be an integer greater than $\alpha_{i}$, $i=1,2$. Now Young's inequality implies $f \equiv f_{1} * f_{2}$ is in $L^{p}\left(\mathbf{R}^{n+1}\right)$. Moreover,

$$
\Gamma f\left(y_{1}+y_{2}\right)=\Gamma f_{1}\left(y_{1}\right) * \Gamma f_{2}\left(y_{2}\right) \text {. }
$$

Thus,

$$
y^{2 k-\alpha_{1}-\alpha_{2}} D_{y}^{2 k} \Gamma f(y)=y^{k-\alpha_{1}}\left(D_{y}^{k} \Gamma f_{1}\right)\left(\frac{y}{2}\right) * y^{k-\alpha_{2}}\left(D_{y}^{k} \Gamma f_{2}\right)\left(\frac{y}{2}\right)
$$

Thus, Young's inequality shows that

$$
\left\|y^{2 k-\alpha_{1}-\alpha_{2}} D_{y}^{2 k} \Gamma f(y)\right\|_{p} \leqq\left\|y^{k-\alpha_{1}}\left(D_{y}^{k} \Gamma f_{1}\right)\left(\frac{y}{2}\right)\right\|\left\|_{p_{1}}\right\| y^{k-\alpha_{2}}\left(D_{y}^{k} \Gamma f_{2}\right)\left(\frac{y}{2}\right) \|_{p_{2}} .
$$

Applying Hölder's inequality then implies

$$
\left\|y^{2 k-\alpha_{1}-\alpha_{2}} D_{y}^{2 k} \Gamma f\right\|_{p q} \leqq 2^{2 k-\alpha_{1}-\alpha_{2}}\left\|y^{k-\alpha_{1}} D_{y}^{k} \Gamma f_{1}\right\|_{p_{1} q_{1}} \cdot\left\|y^{k-\alpha_{2}} D_{y}^{k} \Gamma f_{2}\right\| p_{2} q_{2} \text {. }
$$

Lemma 13. Let $u$ be a solution of $H u=0$ in $\mathrm{R}_{+}^{n+2}$ and assume $\left\|y^{\beta} u\right\|_{p q}<\infty$ for some $\beta>0$. Then $u$ is bounded in each half space $\mathrm{R}^{n+1} \times[\epsilon, \infty), \epsilon>0$ (i.e., $u \in \mathfrak{H C})$.

Proof. It is not difficult to prove that there exists a constant $C$, depending only on $n$, such that

$$
|u(x, t ; y)| \leqq C y^{-n-3} \int_{\substack{|z| \leq y \\ 0 \leqq r \leq y^{2} \\|\eta| \leqq y / 2}}|u(x-z, t-\tau ; y-\eta)| d z d \tau d \eta
$$

This can be derived by proving it for $y=1$ and then using homogeneity; or cf. [6]. Using Minkowski's integral inequality, it follows that

$$
\|u(y)\|_{p} \leqq C^{\prime} y^{-1} \int_{y / 2}^{3 y / 2}\|u(\eta)\|_{p} d \eta
$$


Thus,

$$
\|u(y)\|_{p} \leqq C^{\prime \prime} y^{-\beta} \int_{y / 2}^{3 y / 2} \eta^{\beta}\|u(\eta)\|_{p} \frac{d \eta}{\eta},
$$

and Hölder's inequality implies $\|u(y)\|_{p} \leqq c y^{-\beta}$. The results of Section 2 show that the conclusion of the lemma now follows.

Lemma 14. Let $v$ be a solution of $H v=0$ in $\mathrm{R}^{n+2}$. Let $0<\alpha<k, k$ an integer, and suppose there exist $A>0$ and $y_{0}>0$ such that

$$
\begin{aligned}
\left\|y^{k-\alpha} D_{y}^{k} v\right\|_{p q} & \leqq A ; \\
\|v(y)\|_{p} & \leqq A, \quad y_{0} \leqq y<\infty .
\end{aligned}
$$

Then there exists $f \varepsilon \Lambda^{\prime}(\alpha ; p, q)$ such that $v=\Gamma f$; and

$$
\|f\|_{\alpha ; p, q} \leqq C A
$$

where $C$ is a constant depending only on $\alpha, k, y_{0}$, and $n$.

Proof. Note first that Lemma 13 implies $D_{\nu}^{k} v$ is bounded on closed half spaces of $\mathbf{R}_{+}^{n+2}$. Also (3.10) implies $v$ is bounded for $2 y_{0} \leqq y<\infty$. Thus, Taylor's theorem implies $v$ itself is bounded on closed half spaces on $\mathbf{R}_{+}^{n+2} ;$ i.e., $v \varepsilon \mathcal{F}$, and thus $D_{y} v \varepsilon \mathfrak{H C}_{0}$. Suppose $0<\alpha<1$. Then Lemma 10 implies

$$
\begin{aligned}
\left\|y^{1-\alpha} D_{y} v\right\|_{p q} & \leqq(1-\alpha)^{-1} \cdots(k-1-\alpha)^{-1}\left\|y^{k-\alpha} D_{y}^{k} v\right\|_{p q} \\
& \leqq C A .
\end{aligned}
$$

Also (2.21) implies that $\left\|D_{y} v(y)\right\|_{p}$ is a non-increasing function of $y$. Therefore, the above inequality implies

$$
\begin{aligned}
C A & \geqq\left[\int_{0}^{y}\left\|\eta^{1-\alpha} D_{y} v(\eta)\right\|_{p}^{a} d \eta / \eta\right]^{1 / a} \\
& \geqq\left\|D_{y} v(y)\right\|_{p}\left[\int_{0}^{y} \eta^{(1-\alpha) \alpha-1} d \eta\right]^{1 / a}=[(1-\alpha) q]^{-1 / a} y^{1-\alpha}\left\|D_{y} v(y)\right\|_{p} .
\end{aligned}
$$

That is, $\left\|D_{y} v(y)\right\|_{p} \leqq C^{\prime} y^{\alpha-1}$.

Now suppose $\beta<1 \leqq \alpha$. Then

$$
\begin{aligned}
{\left[\int_{0}^{2 y_{0}}\left\|y^{k-\beta} D_{y}^{k} v(y)\right\|_{p}^{\alpha} d y / y\right]^{1 / a} } & \leqq\left(2 y_{0}\right)^{\alpha-\beta}\left[\int_{0}^{2 y_{0}}\left\|y^{k-\alpha} D_{y}^{k} v(y)\right\|_{p}^{\alpha} d y / y\right]^{1 / \alpha} \\
& \leqq\left(2 y_{0}\right)^{\alpha-\beta} A .
\end{aligned}
$$

Also, if $y>2 y_{0}$ then an easy generalization of (2.22) implies $\left\|D_{y}^{k} v(y)\right\|_{p} \leqq$ $c y^{-k}\|v(y / 2)\|_{p} \leqq c A y^{-k}$. Thus

$$
\left[\int_{2 y_{0}}^{\infty}\left\|y^{k-\beta} D_{y}^{k} v(y)\right\|_{p}^{\alpha} d y / y\right]^{1 / \alpha} \leqq c A\left[\int_{2 y_{0}}^{\infty} y^{-\beta q-1} d y\right]^{1 / \alpha}=c(\beta q)^{-1 / \alpha}\left(2 y_{0}\right)^{-\beta} A
$$

These inequalities together imply that $\left\|y^{k-\beta} D_{y}^{k} v\right\|_{p q} \leqq C^{\prime \prime} A$. Thus, the hypothesis 
(3.9) holds with $\alpha$ replaced by $\beta<1$, so the first part of the argument yields an inequality $\left\|D_{y} v(y)\right\|_{p} \leqq C^{\prime \prime \prime} y^{\beta-1}$.

Thus, in any case there exists $\beta, 0<\beta<1$, such that $\left\|D_{y} v(y)\right\|_{p} \leqq C_{1} y^{\beta-1}$, so that integration gives for $y \leqq y_{0}$

$$
\|v(y)\|_{p} \leqq\left\|v\left(y_{0}\right)\right\|_{p}+\int_{y}^{y_{0}}\left\|D_{\eta} v(\eta)\right\|_{p} d \eta \leqq C_{2} A
$$

and also $\left\|v\left(y_{1}\right)-v\left(y_{2}\right)\right\|_{p} \rightarrow 0$ as $y_{1}, y_{2} \rightarrow 0$. Thus $f \varepsilon L^{p}\left(\mathbf{R}^{n+1}\right)$ exists such that $v(y) \rightarrow f$ in $L^{p}$ as $y \rightarrow 0$. Moreover, Lemmas 6 and 8 imply $v=\Gamma f$, and $\|f\|_{p} \leqq C_{2} A$. Clearly, $f \varepsilon \Lambda^{\prime}(\alpha ; p, q)$.

Lemma 15. If $\alpha>0$, then $D_{x_{j}}: \Lambda^{\prime}(\alpha+1 ; p, q) \rightarrow \Lambda^{\prime}(\alpha ; p, q)$ continuously. Also, $D_{t}: \Lambda^{\prime}(\alpha+2 ; p, q) \rightarrow \Lambda^{\prime}(\alpha ; p, q)$ continuously.

Proof. Let $f \varepsilon \Lambda^{\prime}(\alpha+1 ; p, q)$. Let $v=D_{x_{i}} \Gamma f$. Then $H v=0$ and

$$
\|v(y)\|_{p} \leqq\left\|D_{x_{i}} \Gamma(y)\right\|_{1}\|f\|_{p} \leqq c y^{-1}\|f\|_{p} \leqq c\|f\|_{p}, y \geqq 1 .
$$

Also if $k>\alpha$ then Theorem 2 implies

$$
\begin{aligned}
\left\|y^{k-\alpha} D_{y}^{k} v\right\|_{p q}= & \left\|y^{k+1-(\alpha+1)} D_{y}^{k} D_{x_{j}} \Gamma f\right\|_{p q} \\
& \leqq c\left\|y^{k+1-(\alpha+1)} D_{y}^{k+1} \Gamma f\right\|_{p q} \leqq c\|f\|_{\alpha+1 ; p, q} .
\end{aligned}
$$

Thus Lemma 14 implies $v=\Gamma g$, where $g \varepsilon \Lambda^{\prime}(\alpha ; p, q)$, $\|g\|_{\alpha ; p, \boldsymbol{q}}, \leqq C\|f\|_{\alpha+1 ; p, \boldsymbol{q}}$. Finally it is easily checked that $g=D_{x_{i}} f$ in the distribution sense. A similar proof gives the result for $D_{t}$.

Theorem 4. Suppose $f \varepsilon L^{p}\left(\mathbf{R}^{n+1}\right)$ and $\alpha>0$. Then a necessary and sufficient condition that $f \varepsilon \Lambda^{\prime}(\alpha+2 ; p, q)$ is that $\partial f / \partial x_{i} \varepsilon \Lambda^{\prime}(\alpha+1 ; p, q)$ for $1 \leqq j \leqq n$ and $\partial f / \partial t \varepsilon \Lambda^{\prime}(\alpha ; p, q)$. Moreover the norm $\|f\|_{\alpha+2 ; p, \alpha}$ is equivalent to

$$
\sum_{i=1}^{n}\left\|\partial f / \partial x_{i}\right\|_{\alpha+1 ; p, q}+\|\partial f / \partial t\|_{\alpha ; p, q}+\|f\|_{p} .
$$

Proof. The necessity is a trivial consequence of Lemma 15. Conversely, assume (3.11) is finite and let $u=\Gamma f$. Then $\partial u / \partial x_{i}=\Gamma\left(\partial f / \partial x_{i}\right)$ and $\partial u / \partial t=\Gamma(\partial f / \partial t)$, so that if $k$ is an integer larger than $\alpha+2$,

$$
\left\|y^{k-\alpha-2} D_{y}^{k-1} D_{x_{j}} u\right\|_{p q}=\left\|y^{k-1-(\alpha+1)} D_{y}^{k-1} \Gamma D_{x_{i}} f\right\|_{p_{q}} \leqq c\left\|D_{x_{j}} f\right\|_{\alpha+1 ; p, q},
$$

and likewise

$$
\left\|y^{k-\alpha-1} D_{y}^{k-1} D_{t} u\right\|_{p q} \leqq c\left\|D_{t} f\right\|_{\alpha ; p, q} .
$$

Thus (2.22) implies $\left\|y^{k-\alpha-2} D_{v}^{k} u\right\|_{p q}<\infty$, so that $f \varepsilon \Lambda^{\prime}(\alpha+2 ; p, q)$.

Theorem 5. There is a continuous inclusion $\Lambda^{\prime}\left(\alpha_{1} ; p_{1}, q_{1}\right) \subset \Lambda^{\prime}\left(\alpha_{2} ; p_{2}, q_{2}\right)$ if $p_{1} \leqq p_{2}$ and $\alpha_{1}-(n+2) / p_{1}>\alpha_{2}-(n+2) / p_{2}$ or if $p_{1} \leqq p_{2}$, $\alpha_{1}-(n+2) / p_{1}=\alpha_{2}-(n+2) / p_{2}$, and $q_{1} \leqq q_{2}$. 
Proof. The proof exactly follows the outline in [15] except for a slight change in the case $p_{1} \leqq p_{2}, \alpha_{1}-(n+2) / p_{1}=\alpha_{2}-(n+2) / p_{2}, q_{1}=q_{2}=q$. Let $k>\alpha_{1}$ be an integer, and let $f \varepsilon \Lambda^{\prime}\left(\alpha_{1} ; p_{1}, q\right), u=\Gamma f$. Now Young's inequality shows

$$
\|u(y)\|_{p_{\mathbf{s}}} \leqq\|f\|_{p_{1}}\|\Gamma(y)\|_{r},
$$

where $1 / p_{2}=1 / p_{1}+1 / r-1$. By (2.5) this implies

$$
\|u(y)\|_{p_{2}} \leqq c\|f\|_{p_{1}}, y \geqq 1 .
$$

Next (2.5) again implies

$$
\begin{aligned}
\left\|y^{k-\alpha_{2}} D_{y}^{k} u(y)\right\|_{p_{2}} & \leqq\left\|y^{k-\alpha_{2}}\left(D_{y}^{k} u\right)(y / 2)\right\|_{p_{1}}\|\Gamma(y / 2)\|_{r} \\
& \leqq c\left\|y^{k-\alpha_{2}}\left(D_{\nu}^{k} u\right)(y / 2)\right\|_{p_{2}} y^{-(n+2) / r^{\prime}}=c\left\|y^{k-\alpha_{1}}\left(D_{y}^{k} u\right)(y / 2)\right\|_{p_{1}} .
\end{aligned}
$$

Thus,

$$
\left\|y^{k-\alpha_{2}} D_{y}^{k} u\right\|_{p_{2} Q} \leqq c\|f\|_{\alpha_{1} ; p_{1}, q},
$$

and this inequality together with (3.12) shows that Lemma 14 implies $f \varepsilon \Lambda^{\prime}\left(\alpha_{2} ; p_{2}, q\right)$.

4. Parabolic potentials. The analog of the Bessel potential, [2], [15], will now be discussed. A general treatment of potentials of this type with arbitrary generalized homogeneity has been given [13], but the properties to be derived here are rather special.

Definition. For all complex $\alpha$, the tempered distribution $\mathcal{G}^{\alpha}$ on $\mathbf{R}^{n+1}$ is defined by the formula for its Fourier transform:

$$
\widehat{\mathcal{S}^{\alpha}}(\xi, \tau)=\left(1+|\xi|^{2}+i \tau\right)^{-\alpha / 2}
$$

Here the function $\left(1+|\xi|^{2}+i \tau\right)^{-\alpha / 2}$ is defined to be $\exp \left\{-\alpha / 2 \log \left(1+|\xi|^{2}+i \tau\right)\right\}$, where the branch of the logarithm which is real for real arguments is chosen. It turns out that for $\operatorname{Re} \alpha>0, \mathcal{G}^{\alpha}$ can be written down explicity:

Lemma 16. For $\operatorname{Re} \alpha>0, \mathcal{G}^{\alpha} \varepsilon L^{1}\left(\mathbf{R}^{n+1}\right)$, and

$$
\mathcal{S}^{\alpha}(x, t)= \begin{cases}(4 \pi)^{-n / 2} \Gamma(\alpha / 2)^{-1} t^{(\alpha-n) / 2-1} \exp \left\{-t-|x|^{2} / 4 t\right\}, & t>0, \\ 0, & t<0 .\end{cases}
$$

Proof. It is well known that for $a>0$

$$
\lim _{M \rightarrow \infty} \int_{-M}^{M}(a+i \tau)^{-\alpha / 2} e^{i t \tau} d \tau=\frac{2 \pi}{\Gamma(\alpha / 2)} \begin{cases}e^{-t a} t^{\alpha / 2-1}, & t>0, \\ 0, & t<0 .\end{cases}
$$

Thus, the inverse Fourier transform of $\left(1+|\xi|^{2}+i \tau\right)^{-\alpha / 2}$ is for $t>0$

$(2 \pi)^{-n} \int_{\mathbf{R}^{n}} \Gamma(\alpha / 2)^{-1} e^{-t(1+|\xi| 2)} t^{\alpha / 2-1} e^{i x \cdot \xi} d \xi$ 


$$
\begin{aligned}
& =\Gamma(\alpha / 2)^{-1} t^{(\alpha-n) / 2-1} e^{-t}(2 \pi)^{-n} \int_{\mathbf{R}^{n}} e^{-|\xi|^{2}} e^{i x t-1 / 2 \cdot \xi} d \xi \\
& =\Gamma(\alpha / 2)^{-1} t^{(\alpha-n) / 2-1} e^{-t} 2^{-n} \pi^{-n / 2} e^{-|x|^{2 / 4 t}}
\end{aligned}
$$

and this proves the result.

Lemma 17. For $0<\operatorname{Re} \alpha-(n+2) / p^{\prime}<\min (1, \max (n, 1) / p), \mathcal{G}^{\alpha} \varepsilon \Lambda^{\prime}$ $\left(\operatorname{Re} \alpha-(n+2) / p^{\prime} ; p, \infty\right)$.

Proof. Let $\beta=\operatorname{Re} \alpha-(n+2) / p^{\prime}$. Then the mean value theorem implies that for $|z|<|x| / 2, t>0$,

$$
\begin{aligned}
\left|\mathcal{G}^{\alpha}(x-z, t)-\mathcal{G}^{\alpha}(x, t)\right| & \leqq c t^{1 / 2\left(\beta+(n+2) / p^{\prime}-n\right)-1}|x| t^{-1}|z| \exp \left\{-|x|^{2} / 16 t\right\} \\
& \leqq c t^{1 / 2\left(\beta+(n+2) / p^{\prime}-n-3\right)}|z| \exp \left\{-|x|^{2} / 17 t\right\}
\end{aligned}
$$

Thus,

$$
\begin{aligned}
\| S^{\alpha}(\cdot-z, t)-\mathcal{G}^{\alpha}(\cdot, & t) \|_{p}^{p} \\
& \leqq c^{p}|z|^{p} \int_{|x|>2|z|} t^{p / 2\left(\beta+(n+2) / p^{\prime}-n-3\right)} \exp \left\{-p|x|^{2} / 17 t\right\} d x \\
& +c^{p} \int_{|x|<3|z|} t^{p / 2\left(\beta+(n+2) / p^{\prime}-n-2\right)} \exp \left\{-p|x|^{2} / 4 t\right\} d x \\
& =c^{p}|z|^{p} \int_{|x|>2|z| t-1 / 2} t^{p / 2(\beta-(n+2) / p-1)+n / 2} \exp \left\{-p|x|^{2} / 17\right\} d x \\
& +c^{p} \int_{|x|<3|z| t-1 / 2} t^{p / 2(\beta-(n+2) / p)+n / 2} \exp \left\{-p|x|^{2} / 4\right\} d x
\end{aligned}
$$

Setting $t=|z|^{2} \tau$ then gives

$$
\begin{aligned}
\| \tau_{z, 0} \mathcal{S}^{\alpha}-\mathcal{S}^{\alpha}||_{p}^{p} \leqq c^{p}|z|^{p} \int_{0}^{\infty}|z|^{p(\beta-1)} \tau^{p / 2(\beta-1)-1} d \tau \int_{|x|>2 \tau^{-1 / 3}} \exp \left\{-p|x|^{2} / 17\right\} d x \\
+c^{p} \int_{0}^{\infty}|z|^{p \beta} \tau^{p \beta / 2-1} d \tau \int_{|x|<3 \tau^{-1 / s}} \exp \left\{-p|x|^{2} / 4\right\} d x
\end{aligned}
$$

and one then easily checks that

$$
|z|^{-p \beta}\left\|\tau_{z, 0} \mathcal{G}^{\alpha}-\mathcal{G}^{\alpha}\right\|_{p}^{p} \leqq c^{p} .
$$

The first integral in the above estimate is finite since $\beta<1$, the second since $p \beta<n$. (Note that if $n=0$ there is no condition (4.1) to be checked.)

Next, for $|s|<t / 2$, the mean value theorem implies

$$
\left|\mathcal{G}^{\alpha}(x, t-s)-\mathcal{G}^{\alpha}(x, t)\right| \leqq c t^{1 / 2\left(\beta+(n+2) / p^{\prime}-n\right)-2}|s| \exp \left\{-|x|^{2} / 9 t\right\},
$$

so that

$$
\left\|\mathcal{G}^{\alpha}(\cdot, t-s)-\mathcal{G}^{\alpha}(\cdot, t)\right\|_{p}^{p} \leqq c^{p} t^{p / 2(\beta-(n+2) / p+2)-2 p+n / 2}|s|^{p}
$$


For $|s| \geqq t / 2$,

$$
\begin{aligned}
\left\|\mathcal{G}^{\alpha}(\cdot, t-s)-\mathcal{G}^{\alpha}(\cdot, t)\right\|_{p}^{p} \leqq & c^{p}(t-s)^{p / 2\left(\beta+(n+2) / p^{\prime}-n\right)-p+n / 2} \\
& +c^{p} t^{p / 2\left(\beta+(n+2) / p^{\prime}-n\right)-p+n / 2}
\end{aligned}
$$

where these terms are treated as zero if $t-s<0$ or $t<0$, respectively. Thus, combining these estimates gives

$$
\begin{aligned}
\left\|\tau_{0,8} \mathcal{S}^{\alpha}-\mathcal{G}^{\alpha}\right\|_{p}^{p} & \leqq c^{p} \int_{2|s|}^{\infty} t^{p(\beta / 2-1)-1}|s|^{p} d t+c^{p} \int_{0}^{3|s|} t^{p \beta / 2-1} d t \\
& \leqq c^{p}|s|^{p(\beta / 2)} .
\end{aligned}
$$

Combining this estimate with (4.1) gives the result that in the notation of Theorem $3, B<\infty$. To conclude that $\mathcal{G}^{\alpha} \varepsilon \Lambda^{\prime}(\beta ; p, \infty)$, it must finally be shown that $\mathcal{G}^{\alpha} \varepsilon L^{p}$. But

$$
\left\|\mathrm{S}^{\alpha}\right\|_{p}^{p}=c^{p} \int_{0}^{\infty} t^{p / 2\left(\beta+(n+2) / p^{\prime}-n\right)-p+n / 2} e^{-p t} d t<\infty
$$

since the exponent of $t$ is $p \beta / 2-1>-1$.

Theorem 6. For $0<\operatorname{Re} \alpha-(n+2) / p^{\prime}, \mathcal{S}^{\alpha} \varepsilon \Lambda^{\prime}\left(\operatorname{Re} \alpha-(n+2) / p^{\prime} ; p, \infty\right)$.

Proof. This type of argument can be found in [15]. The important observation is that $\mathcal{G}^{\alpha} * \mathcal{G}^{\beta}=\mathcal{G}^{\alpha+\beta}$, so that Lemma 12 is directly applicable. Obviously, it can be assumed in the proof that all the indices $\alpha$ for $\mathcal{G}^{\alpha}$ are real. Then since $\mathcal{G}^{\alpha / 2} \varepsilon \Lambda^{\prime}(\alpha / 2-(n+2) / 2 ; 2, \infty)$ for $0<\alpha / 2-(n+2) / 2<\min \left(1,2^{-1} \max (n, 1)\right)$, it follows from Lemma 12 that $\mathcal{G}^{\alpha} \varepsilon \Lambda^{\prime}(\alpha-n-2 ; \infty, \infty)$ for such $\alpha$. Combining this with Lemma 17 for $p<\infty$, it follows that for all $p \varepsilon[1, \infty]$ there exists $\epsilon(p)>0$ such that

$$
\mathcal{G}^{\beta} \varepsilon \Lambda^{\prime}\left(\beta-(n+2) / p^{\prime} ; p, \infty\right) \text { if } 0<\beta-(n+2) / p^{\prime}<\epsilon(p) .
$$

Now suppose $0<\alpha-(n+2) / p^{\prime}$, and write $\alpha=\beta+(k-1) \gamma$, where $0<\beta-$ $(n+2) / p^{\prime}<\epsilon(p), k \geqq 1$ is an integer, $0<\gamma<1$. Then $\mathcal{G}^{\alpha}=\mathcal{G}^{\beta} * \mathcal{G}^{\gamma} * \ldots * \mathcal{G}^{\gamma}$, where there are $k$ factors. By Lemma $17, g^{\gamma} \varepsilon \Lambda^{\prime}(\gamma ; 1, \infty)$, and by the above considerations, $\mathcal{G}^{\beta} \varepsilon \Lambda^{\prime}\left(\beta-(n+2) / p^{\prime} ; p, \infty\right)$. Thus, Lemma 12 implies that $\mathcal{G}^{\alpha} \varepsilon \Lambda^{\prime}\left(\beta-(n+2) / p^{\prime}+(k-1) \gamma ; p, \infty\right)$, as required.

Now a linear transformation associated with $\mathrm{g}^{\alpha}$ is defined by the formula

$$
\mathscr{J}_{\alpha} f=\mathcal{S}^{\alpha} * f, f \varepsilon \mathcal{S}^{\prime}\left(\mathbf{R}^{n+1}\right) .
$$

For all $\alpha \varepsilon \mathbf{C}$ it follows that $\mathscr{J}_{\alpha}: \mathcal{S}^{\prime}\left(\mathbf{R}^{n+1}\right) \rightarrow S^{\prime}\left(\mathbf{R}^{n+1}\right)$.

Theorem 7. If $\alpha>0$ and $\alpha+\beta>0$, then $\mathcal{J}_{\beta}: \Lambda^{\prime}(\alpha ; p, q) \rightarrow \Lambda^{\prime}(\alpha+\beta ; p, q)$ is an isomorphism of topological linear spaces.

Proof. Since $\mathscr{J}_{\beta} \mathcal{J}_{-\beta}$ is the identity on $\mathcal{S}^{\prime}\left(\mathbf{R}^{n+1}\right)$ it suffices to prove that $\mathscr{J}_{\beta}$ maps $\Lambda^{\prime}(\alpha ; p, q)$ continuously into $\Lambda^{\prime}(\alpha+\beta ; p, q)$. If $\beta>0$ this is an immediate consequence of the fact that $\mathscr{g}_{\beta} f=\mathcal{G}^{\beta} * f$ and Theorem 6 and Lemma 12. If $\beta<0$ 
then we use the relation $\mathfrak{d}_{\beta}=\mathcal{J}_{-2 m} \mathfrak{d}_{\beta+2 m}$, where $m$ is an integer such that $\beta+2 m>0$. Then $\mathscr{g}_{\beta+2 m}$ maps $\Lambda^{\prime}(\alpha ; p, q)$ into $\Lambda^{\prime}(\alpha+\beta+2 m ; p, q)$ continuously. And the definition of $\mathrm{g}^{-2 m}$ shows that

$$
\mathcal{J}_{-2 m}=\left(1-\Delta+\frac{\partial}{\partial t}\right)^{m} \text {. }
$$

Thus, Lemma 15 and Theorem 5 imply that $\mathcal{J}_{-2 m}$ maps $\Lambda^{\prime}(\alpha+\beta+2 m ; p, q)$ into $\Lambda^{\prime}(\alpha+\beta ; p, q)$ continuously.

5. Solution of the classification problem for $n=0$. The results of Section 3 allow one explicity to describe $\Lambda^{\prime}(\alpha ; p, q)$ for most $\alpha>0$. That is, a characterization of $\Lambda^{\prime}(\alpha ; p, q)$ can be given without referring to integral operators or other extraneous tools, but by using only derivatives or difference quotients of functions. Theorem 4 shows that if $\Lambda^{\prime}(\alpha ; p, q)$ is so characterized for $0<\alpha \leqq 2$, then the characterization extends to all $\alpha>2$ simply by considering the behavior of derivatives of functions. And Theorem 3 achieves a description of $\Lambda^{\prime}(\alpha ; p, q)$ for $0<\alpha<2$. However, at this time a description of $\Lambda^{\prime}(2 ; p, q)$ is not known. The basic difficulty is due to the fact that some condition on a second $t$ difference of a function $f$ is probably necessary for $f$ to belong to $\Lambda^{\prime}(2 ; p, q) ; e . g$. (5.1). But it is difficult to use such information to derive information about $D_{v}^{k} \Gamma f$ since the kernel $\Gamma$ fails to be an even function of $t$, and therefore the presence of $f(t-s)$ in the expression for $D_{y}^{k} \Gamma f$ does not insure that $f(t+s)$ can be introduced as well. In this connection see the italicized remark following (3.5). Some information can be derived in the other direction, however, as shown in the following result.

Lemma 18. Let $1<\alpha<4$ and suppose $f \varepsilon \Lambda^{\prime}(\alpha ; p, q)$. Then $D_{x_{j}} f \varepsilon \Lambda^{\prime}(\alpha-1 ; p, q)$ and the $L^{a}(d s /|s|)$ - norm of the function

$$
\frac{\left\|\tau_{0, s} f-2 f+\tau_{0,-s} f\right\|_{p}}{|s|^{\alpha / 2}}
$$

is finite.

Proof. The statement about $D_{x_{i}} f$ is obvious from Lemma 15 . The other statement can be proved by a technique similar to that used in the proof of the estimate for $B_{1}$ in Theorem 3 and the details need not be given here.

A reasonable conjecture might be that the converse of Lemma 18 is also valid. This would of course solve the classification problem for $\Lambda^{\prime}(2 ; p, q)$. However, the converse is valid at least for $n=0$, and the proof will now be given. First, some lemmas will be given. In the rest of this section the functions will be defined on $\mathbf{R}$.

Using the terminology of [15] let $0<\alpha<2$ and let $\Lambda(\alpha ; p, q)$ be the subspace of $L^{p}(\mathbf{R})$ for which the norm

$$
\|f\|_{p}+L^{\alpha}\left(\frac{d s}{|s|}\right)-\text { norm of }|s|^{-\alpha}\left\|\tau_{s} f-2 f+\tau_{-s} f\right\|_{p}
$$


is finite. Here $\left(\tau_{s} f\right)(t)=f(t-\mathbf{s})$. Also define $J_{\beta}: \mathfrak{S}^{\prime}(\mathbf{R}) \rightarrow \mathfrak{S}^{\prime}(\mathbf{R})$ by the formula for Fourier transforms in R:

$$
\widehat{J_{\beta} f}=\left(1+\tau^{2}\right)^{-\beta / 2} \hat{f} .
$$

Then $J_{\beta}$ is an isomorphism of $\Lambda(\alpha ; p, q)$ onto $\Lambda(\alpha+\beta ; p, q)$ if $\alpha$ and $\alpha+\beta$ are in $(0,2)$, as a result of Theorem 5 of [15]. Also $\Lambda(\alpha ; p, q)$ can be defined for all $\alpha>0$ and the same result holds for $\alpha>0, \alpha+\beta>0$.

Lemma 19. Let $\gamma \varepsilon \mathrm{R}$ and let

$$
F(\tau)=\left(\frac{1-i \tau}{1+i \tau}\right)^{\gamma}, \quad \tau \varepsilon \mathbf{R},
$$

where the principal branch of the power is taken. Then there exists a totally finite Borel measure $\mu$ on $\mathbf{R}$ such that in the sense of Fourier transforms in $\mathrm{S}^{\prime}$

$$
F=\frac{\sin \gamma \pi}{\pi} \hat{T}+\hat{\mu},
$$

where $T \varepsilon \mathcal{S}^{\prime}$ is defined as a truncated Hilbert transform:

$$
\begin{aligned}
T(\varphi) & =P \int_{-1}^{1} \frac{\varphi(t)}{t} d t \\
& \equiv \lim _{\epsilon \rightarrow 0} \int_{\epsilon<|t|<1} \frac{\varphi(t)}{t} d t, \quad \varphi \varepsilon \delta .
\end{aligned}
$$

Proof. By definition $F(\tau)=\exp \left\{-2 i \gamma \tan ^{-1} \tau\right\}$, where $-\pi / 2<\tan ^{-1} \tau<\pi / 2$. Thus $F=F_{1}+\cos \gamma \pi-i F_{2}$, where

$$
\begin{aligned}
& F_{1}(\tau)=\cos \left(2 \gamma \tan ^{-1} \tau\right)-\cos \gamma \pi, \\
& F_{2}(\tau)=\sin \left(2 \gamma \tan ^{-1} \tau\right) .
\end{aligned}
$$

One can easily check that $F_{1}=\hat{f}$, where $f \varepsilon L^{1}(\mathrm{R})$, since on the one hand $F_{1} \varepsilon L^{2}$ and on the other hand all derivatives of $F_{1}$ are in $L^{1}$.

Next, if $H$ is the Hilbert transform:

$$
H(\varphi)=\frac{i}{\pi} P \int_{-\infty}^{\infty} \frac{\varphi(t)}{t} d t
$$

then it is well known that $\hat{H}=\operatorname{sgn} \tau$. Thus, since $F_{2}-\sin \gamma \pi \operatorname{sgn} \tau$ is in $L^{2}$, there exists $g \& L^{2}$ such that

$$
F_{2}=\sin \gamma \pi \hat{H}+\hat{g} .
$$

On the other hand, $F_{2}^{\prime}=2 \gamma \cos \left(2 \gamma \tan ^{-1} \tau\right)\left(1+\tau^{2}\right)^{-1}$. Since the Fourier transform of $e^{-|t|}$ is $2\left(1+\tau^{2}\right)^{-1}$, this can be written

$$
F_{2}^{\prime}=\gamma \cos \gamma \pi e^{-|t|}+2 \gamma \frac{\cos \left(2 \gamma \tan ^{-1} \tau\right)-\cos \gamma \pi}{1+\tau^{2}}
$$


The second term on the right hand side of (5.3) can be easily seen to have an inverse Fourier transform which has a bounded continuous derivative and which decays as $t \rightarrow \infty$ faster than any power of $t$. Thus, if $\breve{F}_{2}$ denotes the inverse Fourier transform of $F_{2},(5.3)$ implies

$$
\text { - it } \check{F}_{2}=h \text {, }
$$

where $h$ is uniformly Lipschitz continuous of order 1 and decays as $t \rightarrow \infty$ faster than any power of $t$. Furthermore,

$$
\begin{aligned}
h(0) & =\left(F_{2}^{\prime}\right)^{\swarrow}(0)=\frac{1}{2 \pi} \int_{-\infty}^{\infty} F_{2}^{\prime}(\tau) d \tau \\
& =\frac{1}{\pi} F_{2}(\infty)=\frac{\sin \gamma \pi}{\pi} .
\end{aligned}
$$

Dividing (5.4) by - it gives

$$
\check{F}_{2}=i \frac{h}{t}+S,
$$

where $h / t$ is a principal value distribution and $S$ is a distribution with support equal to the origin (or else $S=0$ ). It is well known that $S$ is a finite linear combination of the Dirac measure $\delta$ and its derivatives. But (5.2) and (5.5) together imply that

$$
S=\sin \gamma \pi H+g-i \frac{h}{t}
$$

As the right side of (5.6) has a continuous extension to $L^{2}$ but none of the distributions $\delta^{(k)}$ does, (5.6) implies $S=0$. Thus, combining results gives

$$
F=\hat{f}+\cos \gamma \pi+(h / t)^{\wedge} .
$$

Thus, the lemma follows with

$$
\mu=f+(\cos \gamma \pi) \delta+ \begin{cases}\frac{h(t)-h(0)}{t}, & |t|<1 \\ \frac{h(t)}{t}, & |t|>1 .\end{cases}
$$

Lemma 20. Let $F(\tau)$ be as in Lemma 19. Let $\Phi: \mathrm{s}^{\prime} \rightarrow \mathrm{s}^{\prime}$ be defined by the formula

$$
\widehat{\Phi f}=F \hat{f}, \quad f \varepsilon S^{\prime} .
$$

Then $\Phi$ is a continuous linear transformation from $\Lambda(\alpha ; p, q)$ to $\Lambda(\alpha ; p, q)$.

Proof. By Lemma 19, $\Phi f=a T * f+\mu * f$. As $\mu$ is a totally finite measure, the mapping $f \rightarrow \mu * f$ is a continuous translation invariant operator from $L^{p}$ to $L^{p}$, $1 \leqq p \leqq \infty$. It is trivial to verify that such an operator maps $\Lambda(\alpha ; p, q)$ continuously into itself. The corresponding result for $f \rightarrow T * f$ is contained essentially 
in [16], where it is remarked that singular integral operators behave well locally in this respect. The explicit verification is a standard exercise in proving that singular integral operators locally preserve Hölder continuity, and therefore the proof is omitted.

Theorem 8. For $\alpha>0, \Lambda^{\prime}(2 \alpha ; p, q)=\Lambda(\alpha ; p, q)$.

Proof. The explicit characterization of Theorem 3 shows that this relation holds for $0<\alpha<1$. And Theorem 4 together with the corresponding result for $\Lambda(\alpha ; p, q)$ shows that if the relation holds for $0<\alpha \leqq 1$, then it holds for $\alpha>0$. So it suffices to prove $\Lambda^{\prime}(2 ; p, q)=\Lambda(1 ; p, q)$. Let $0<\beta<1$. Then it is easily seen that Lemma 20 implies $\mathcal{J}_{-2 \beta} J_{\beta}$ is an isomorphism of $\Lambda(\alpha ; p, q)$ onto itself. For

$$
\begin{aligned}
\widehat{\mathcal{J}_{-2 \beta} J_{\beta} f} & =(1+i \tau)^{\beta}\left(1+\tau^{2}\right)^{-\beta / 2} \hat{f} \\
& =\left(\frac{1-i \tau}{1+i \tau}\right)^{-\beta / 2} \hat{f},
\end{aligned}
$$

and the inverse is of the same form. Thus,

$$
\begin{aligned}
\Lambda(1 ; p, q) & =J_{\beta} \Lambda(1-\beta ; p, q) \\
& =g_{2 \beta}\left(\mathcal{J}_{-2 \beta} J_{\beta}\right) \Lambda(1-\beta ; p, q) \\
& =g_{2 \beta} \Lambda(1-\beta ; p, q) \\
& =g_{2 \beta} \Lambda^{\prime}(2-2 \beta ; p, q) \\
& =\Lambda^{\prime}(2 ; p, q) .
\end{aligned}
$$

Added in proof. It has now been shown by the author that the converse of Lemma 18 is valid even for $n>0$. A technique is used which exploits the validity of Theorem 8 for $n=0$.

Appendix. It is frequently necessary in this paper to consider integrals of functions on $\mathbf{R}^{n}$ with respect to measures other than Lebesgue measure. Specifically, let $\alpha_{i}>0,1 \leqq i \leqq n$, and consider the measure

$$
\overline{d x}=\frac{d x_{1} \cdots d x_{n}}{\left(\left|x_{1}\right|^{1 / \alpha_{1}}+\cdots+\left|x_{n}\right|^{1 / \alpha_{n}}\right)^{\alpha_{1}+\cdots+\alpha_{n}}} .
$$

This measure has the property that it is invariant with respect to variable transformations of the form $x_{i}=\lambda^{\alpha_{i}} z_{i}, 1 \leqq i \leqq \mathrm{n}$.

Lemma A. If $f \geqq 0$ is a measurable function which is independent of $x_{m+1}, \ldots, x_{n}$, where $1 \leqq m \leqq n$, then

$$
\int f \overline{d x}=C \int f \frac{d x_{1} \cdots d x_{m}}{\left(\left|x_{1}\right|^{1 / \alpha_{1}}+\cdots+\left|x_{m}\right|^{1 / \alpha_{m}}\right)^{\alpha_{1}+\cdots+\alpha_{m}}},
$$

where $C$ depends only on $\alpha_{1}, \ldots, \alpha_{n}, m$, and $n$. 
Proof. Using Fubini's theorem,

$$
\int f \overline{d x}=\int f d x_{1} \cdots d x_{m} \int \frac{d x_{m+1} \cdots d x_{n}}{\left(\left|x_{1}\right|^{1 / \alpha_{1}}+\cdots+\left|x_{n}\right|^{1 / \alpha_{n}}\right)^{\alpha_{1}+\cdots+\alpha_{n}}} .
$$

In the inner integral on the right side set

$$
x_{i}=\left(\left|x_{1}\right|^{1 / \alpha_{1}}+\cdots+\left|x_{m}\right|^{1 / \alpha_{m}}\right)^{\alpha_{j}} z_{j}, \quad m+1 \leqq j \leqq n ;
$$

This integral then becomes

$$
\frac{1}{\left(\left|x_{1}\right|^{1 / \alpha_{1}}+\cdots+\left|x_{m}\right|^{1 / \alpha_{m}}\right)^{\alpha_{1}+\cdots+\alpha_{m}}} C
$$

where

$$
C=\int \frac{d z_{m+1} \cdots d z_{n}}{\left(1+\left|z_{m+1}\right|^{1 / \alpha_{m+1}}+\cdots+\left|z_{n}\right|^{1 / \alpha_{n}}\right)^{\alpha_{1}+\cdots+\alpha_{n}}}
$$

Lemma $B$. If $f \geqq 0$ is a measurable function which depends only on $\left|x_{1}\right|^{1 / \alpha_{1}}+\cdots+\left|x_{n}\right|^{1 / \alpha_{n}}$, then

$$
\int f \overline{d x}=C_{1} \int_{0}^{\infty} f(y) \frac{d y}{y}
$$

where $C_{1}$ depends only on $\alpha_{1}, \cdots, \alpha_{n}$.

Proof. Let $y=\left|x_{1}\right|^{1 / \alpha_{1}}+\cdots+\left|x_{n}\right|^{1 / \alpha_{n}}$, changing $x_{1}$ to $y$. Then

$$
\int f \overline{d x}=2 \alpha_{1} \int_{0}^{\infty} f(y) d y \int_{x_{1}>0} \frac{\left|x_{1}\right|^{1-1 / \alpha_{1}}}{y^{\alpha_{1}+\cdots+\alpha_{n}}} d x_{2} \cdots d x_{n} .
$$

The inner integral can be easily computed by using the change of variables $x_{i}=y^{\alpha_{i}} z_{j}, j>1$; it equals

$$
y^{-1} \int_{\left|z_{2}\right| 1 / \alpha_{2}+\cdots+\left|z_{n}\right| \alpha_{1} / \alpha_{n}<1}\left(1-\left|z_{2}\right|^{1 / \alpha_{2}}-\cdots-\left|z_{n}\right|^{1 / \alpha_{n}}\right)^{\alpha_{1}-1} d z_{2} \cdots d z_{n} .
$$

It is easy to check that this integral converges.

Lemma $C$. If $\varphi(x) \geqq 0$ and $\psi(z) \geqq 0$ are measurable functions for $x \boldsymbol{\varepsilon} \mathbf{R}^{k}$ and $z \boldsymbol{\varepsilon} \mathbf{R}^{n}$, respectively, and if $T$ is the integral transform

$$
T f(x)=\int K(x, z) f(z) d z
$$

where $K$ is a non negative measurable function on $\mathbf{R}^{k} \times \mathbf{R}^{n}$, then a necessary and sufficient condition that $T$ be a bounded operator from

$$
L^{p}\left(\frac{d z}{\psi(z)}\right) \quad \text { to } \quad L^{p}\left(\frac{d x}{\varphi(x)}\right)
$$


for all $p \varepsilon[1, \infty]$ is that

$$
\begin{array}{r}
\int K(x, z) d z \leqq C_{1}, \quad x \varepsilon \mathbf{R}^{k}, \\
\int K(x, z) \frac{\psi(z)}{\varphi(x)} d x \leqq C_{2}, \quad z \varepsilon \mathbf{R}^{n} .
\end{array}
$$

Proof. The first inequality states that $T$ is bounded for $p=\infty$ with norm $\leqq C_{1}$, and the second that $T$ is bounded for $p=1$ with norm $\leqq C_{2}$. The result follows by interpolation.

Lemma $D$ Let $u$ be a function of class $C^{2}$ on $\mathbf{R}^{n}$. Then for any $z \mathbf{\varepsilon} \mathbf{R}^{n}$

$$
\|u(\cdot+z)-2 u+u(\cdot-z)\|_{p} \leqq \sum_{i, j=1}^{n}\left|z_{i}\right|\left|z_{j}\right|\left\|D_{x_{i x} x_{j}} u\right\|_{p} .
$$

Proof. Let $a(t)=u(x+t z)$. Then Taylor's theorem gives

$$
a(1)-2 a(0)+a(-1)=\int_{-1}^{1}(1-|s|) a^{\prime \prime}(s) d s .
$$

Thus,

$$
u(x+z)-2 u(x)+u(x-z)=\int_{-1}^{1}(1-|s|) \sum_{i, j=1}^{n} u_{x_{i x} x_{i}}(x+s z) z_{i} z_{i} d s .
$$

and Minkowski's integral inequality gives the result.

Lemma $E$ (HARDY). Let $\alpha>0, f \geqq 0$ a measurable function on $(0, \infty)$, and

$$
\begin{aligned}
& F_{1}(t)=t^{-\alpha} \int_{0}^{t} f(s) d s \\
& F_{2}(t)=t^{\alpha} \int_{t}^{\infty} f(s) d s .
\end{aligned}
$$

Then

$$
\begin{aligned}
& \left\|F_{1}\right\|_{L^{\alpha}(d t / t)} \leqq \alpha^{-1}\left\|t^{1-\alpha} f\right\|_{L^{\alpha}(d t / t)} \\
& \left\|F_{2}\right\|_{L^{\alpha}(d t / t)} \leqq \alpha^{-1}\left\|t^{1+\alpha} f\right\|_{L^{\alpha}(d t / t)} .
\end{aligned}
$$

See [18], p. 20.

\section{References}

[1] G. Arnese, Maggiorazioni in $L^{p}$ dei potenziali relativi all' equazione del calore, Ricerche Mat., 13 (1964) 147-191.

[2] N. AronszaJn \& K. T. Smith, Thcory of Bessel potentials, I, Ann. Inst. Fourier, 11 (1961) $385-475$.

[3] R. E. Edwards \& E. Hewitr, Pointwise limits for sequences of convolution operators, Acta Math., 113 (1965) 181-218.

[4] E. B. FABES \& N. M. RIvrèRE, Singular integrals with mixed homogeneity, Stud. Math., 27 (1966) 19-38. 
[5] A. Friedman, Partial Differential Equations of Parabolic Type, Prentice Hall, Inc., Englewood Cliffs, N. J., 1964.

[6] J. R. HAtTemer, Boundary behavior of temperatures, I, Stud. Math., 25 (1964) 111-155.

[7] B. Jessen, J. Marcinkinwicz, \& A. Zygmund, Note on the differentiability of multiple integrals, Fund Math., 25 (1935) 217-234.

[8] B. F. Jones, JR., A class of singular integrals, Amer. J. Math., 86 (1964) 441-462.

[9] B. F. Jones, JR., Singular integrals and a boundary value problem for the heat equation, Symp. on Pure Math., 10 (1967) 196-207.

[10] S. M. Nikolskí, On the embedding, continuity, and approximation theorems for differentiable functions in several variables, Uspekhi Mat. Nauk, 16 (1961) 63-114.

[11] L. Nirenberg, A strong maximum principle for parabolic equations, Comm. Pure Appl. Math., 6 (1953) 167-177.

[12] J. PeEtre, On an equivalence theorem of Taibleson, preprint.

[13] C. Sadosky \& M. Cotlar, On quasi-homogeneous Bessel potential operators, Symp. on Pure Math., 10 (1967) 275-287.

[14] L. Schwartz, Théorie des Distributions, vol. I-II, Paris, 1951.

[15] M. H. Tambleson, On the theory of Lipschitz spaces of distributions on Euclidean $n$ space, I. Principal properties, J. Math. Mech., 13 (1964) 407-480.

[16] - II. Translation invariant operators, duality, and interpolation, J. Math. Mech., 14 (1965) 821-840.

[17] - III. Smoothness and integrability of Fourier transforms, smoothness of convolution transforms, J. Math. Mech., 15 (1966) 973-981.

[18] A. Zxamund, Trigonometric Series, vol. I-II, 2d. ed., Cambridge, 1959.

Rice University

Date communicated: December 19, 1967 